\title{
Series Solution of the System of Fuzzy Differential Equations
}

\author{
M. S. Hashemi, ${ }^{1,2}$ J. Malekinagad, ${ }^{2}$ and H. R. Marasi ${ }^{2}$ \\ ${ }^{1}$ Dipartimento di Mathematica e Informatica, Università degli Stueli di Perugia, 06123, Italy \\ ${ }^{2}$ Department of Mathematics and Computer Science, Engineering and Technical Faculty, University of Bonab, Bonab 55517, Iran
}

Correspondence should be addressed to M. S. Hashemi, hashemi_math396@yahoo.com

Received 9 April 2012; Accepted 28 June 2012

Academic Editor: Rustom M. Mamlook

Copyright ( $) 2012$ M. S. Hashemi et al. This is an open access article distributed under the Creative Commons Attribution License, which permits unrestricted use, distribution, and reproduction in any medium, provided the original work is properly cited.

\begin{abstract}
The homotopy analysis method (HAM) is proposed to obtain a semianalytical solution of the system of fuzzy differential equations (SFDE). The HAM contains the auxiliary parameter $\hbar$, which provides us with a simple way to adjust and control the convergence region of solution series. Concept of $\hbar$-meshes and contour plots firstly are introduced in this paper which are the generations of traditional $h$-curves. Convergency of this method for the SFDE has been considered and some examples are given to illustrate the efficiency and power of HAM.
\end{abstract}

\section{Introduction}

In many cases of the modeling real world phenomena, information about the behavior of a dynamical system is uncertain. In order to obtain a more realistic model, we have to take into account these uncertainties. Since 1965, when Zadeh published his pioneering paper [1], hundreds of examples have been supplied where the nature of uncertainty in the behavior of a given system processes is fuzzy rather than stochastic nature. The concept of fuzzy derivative was first introduced by Chang and Zadeh in [2]. It was followed up by Dubois and Prade in [3], who defined and used the extension principle. Other methods have been discussed by Puri and Ralescu in [4] and Goetschel and Voxman in [5]. The initial value problem for fuzzy differential equation (FIVP) has been studied by Kaleva in $[6,7]$ and by Seikkala in $[8]$.

The purpose of this paper is to find the approximate solution of fuzzy differential equations system with the homotopy analysis method (HAM) introduced first by Liao in $1992[9,10]$, that is, analytic approach to get series solutions of various types of linear and nonlinear equations.

Some of numerical methods have been applied to obtain the solution of fuzzy differential equations [11-15]. Sami Bataineh et al. have applied the HAM for systems of ODEs and PDEs in $[16,17]$. Also, recently many types of nonlinear problems solved with HAM by others [18-22].
In Section 2, some basic definitions which will be used later in the paper are provided. In Sections 3 and 4, system of fuzzy differential equations and then basic ideas of HAM applied to these types of equations have been reviewed, respectively. Convergency of HAM for SFDE that shows its reliability has been considered in Section 5. The proposed method is illustrated by solving several examples in Section 6, and finally the conclusion is drawn in Section 7.

\section{Preliminaries}

In this section, the most basic notations used in this paper are introduced.

Definition 1 (See [23]). An arbitrary fuzzy number $u$ in parametric form is an ordered pair $(\underline{u}(r), \bar{u}(r))$ of functions $\underline{u}(r)$, $\bar{u}(r) ; 0 \leq r \leq 1$, which satisfies the following requirements:

(1) $\underline{u}(r)$ is a bounded left-continuous nondecreasing function over $[0,1]$,

(2) $\bar{u}(r)$ is a bounded left-continuous nonincreasing function over $[0,1]$,

(3) $\underline{u}(r) \leq \bar{u}(r), 0 \leq r \leq 1$.

The set of all such fuzzy numbers is represented by $\mathbb{E}^{1}$. 
Remark 2. For arbitrary $u=(\underline{u}(r), \bar{u}(r)), v=(\underline{v}(r), \bar{v}(r))$, and $k \in \mathbb{R}$, we define addition and multiplication by $k$ as

$$
\begin{aligned}
\underline{(u+v)}(r) & =\underline{u}(r)+\underline{v}(r), \quad \overline{(u+v)}(r)=\bar{u}(r)+\bar{v}(r), \\
\underline{k u}(r) & =k \underline{u}(r), \quad \overline{k u}(r)=k \bar{u}(r), \quad \\
\underline{k u}(r) & =k \bar{u}(r), \quad \overline{k u}(r)=k \underline{u}(r), \quad \text { if } k<0 .
\end{aligned}
$$

Definition 3. For arbitrary fuzzy numbers $u, v \in \mathbb{E}^{1}$, we use the distance

$$
D(u, v)=\sup _{0 \leq r \leq 1} \max \{|\bar{u}(r)-\bar{v}(r)|,|\underline{u}(r)-\underline{v}(r)|\},
$$

and it is shown that $\left(\mathbb{E}^{1}, D\right)$ is a complete metric space.

Definition 4. Let $f:[a, b] \rightarrow \mathbb{E}^{1}$, for each partition $P=$ $\left\{t_{0}, \ldots, t_{n}\right\}$ of $[a, b]$ and for arbitrary $\xi_{i} \in\left[t_{i-1}, t_{i}\right], 1 \leq i \leq n$ suppose

$$
R_{p}=\sum_{i=1}^{n} f\left(\xi_{i}\right)\left(t_{i}-t_{i-1}\right)
$$

$$
\Delta:=\max \left\{\left|t_{i}-t_{i-1}\right|, i=1, \ldots, n\right\} .
$$

The definite integral of $f(t)$ over $[a, b]$ is

$$
\int_{a}^{b} f(t) d t=\lim _{\Delta \rightarrow 0} R_{p}
$$

provided that this limit exists in the metric $D$. If the fuzzy function $f(t)$ is continuous in the metric $D$, its definite integral exists and also,

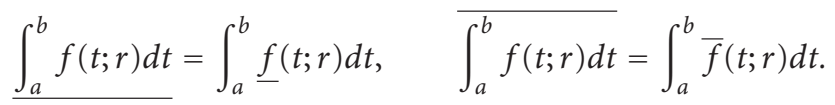

Definition 5. Let $f: \mathbb{R}^{1} \rightarrow \mathbb{E}^{1}$ be a fuzzy function and let $t_{0} \in \mathbb{R}^{1}$. The derivative $f^{\prime}\left(t_{0}\right)$ of $f$ at the point $t_{0}$ is defined by

$$
f^{\prime}\left(t_{0}\right)=\lim _{h \rightarrow 0} \frac{f\left(t_{0}+h\right)-f\left(t_{0}\right)}{h},
$$

provided that this limit, taken with respect to the metric $D$, exists.

The elements $f\left(t_{0}+h\right), f\left(t_{0}\right)$ at the right-hand side of (6) are observed as elements in the Banach space $\mathbb{B}=\mathbb{C}[0,1] \times$ $\mathbb{C}[0,1]$. Thus, if $f\left(t_{0}+h\right)=(\underline{a}, \bar{a})$ and $f\left(t_{o}\right)=(\underline{b}, \bar{b})$, the difference is simply $f\left(t_{0}+h\right)-f\left(t_{o}\right)=(\underline{a}-\underline{b}, \bar{a}-\bar{b})$.

Clearly $\left[f\left(t_{0}+h\right)-f\left(t_{0}\right)\right] / h$ may not be a fuzzy number for all $h$. However, if it approaches $f^{\prime}\left(t_{0}\right)$ (in $\mathbb{B}$ ) and $f^{\prime}\left(t_{0}\right)$ is also a fuzzy number (i.e., in $\mathbb{E}^{1}$ ), this number is the fuzzy derivative of $f(t)$ at $t_{0}$. In this case, if $f=(\underline{f}, \bar{f})$, it can be easily shown that

$$
f^{\prime}\left(t_{0}\right)=\left(\underline{f^{\prime}}\left(t_{0}\right), \bar{f}^{\prime}\left(t_{0}\right)\right),
$$

where $f^{\prime}$ and $\bar{f}^{\prime}$ are the classic derivatives of $\underline{f}$ and $\bar{f}$, respectively.

\section{System of Fuzzy Differential Equations}

In this section, we will review system of fuzzy differential equations of the forms

$$
\begin{gathered}
\sum_{i=0}^{n} A_{i}(t) Y^{(i)}(t)=F(t), \\
Y^{(i)}(0)=G_{i}, \quad i=0, \ldots, n-1,
\end{gathered}
$$

where $t$ is a scaler and $A_{0}(t), A_{1}(t), \ldots, A_{n-1}(t)$ are $s \times s$ matrixes and every component of them is a real function of $t$ and $A_{n}(t)=I_{s}$ denotes the $s \times s$ identity matrix. $Y, G_{i}$, and $F$ are fuzzy $s$-dimensional vectors. The $r$ th component of $Y \in E^{s}$ will be denoted by $y_{r}$, so that we may write

$$
\begin{gathered}
Y=\left[y_{1}(t, r), y_{2}(t, r), \ldots, y_{s}(t, r)\right]^{T}, \\
y_{i}(t, r)=\left(\left(y_{l}\right)_{i}(t, r),\left(y_{u}\right)_{i}(t, r)\right), \quad i=1, \ldots, s, \\
F=\left[f_{1}(t, r), f_{2}(t, r), \ldots, f_{s}(t, r)\right]^{T}, \\
f_{i}(t, r)=\left(\left(f_{l}\right)_{i}(t, r),\left(f_{u}\right)_{i}(t, r)\right), \quad i=1, \ldots, s, \\
G_{i}=\left[g_{i 1}(r), g_{i 2}(r), \ldots, g_{i s}(r)\right]^{T},
\end{gathered}
$$

where

$$
\begin{gathered}
g_{i j}(r)=\left(\left(g_{l}\right)_{i j}(r),\left(g_{u}\right)_{i j}(r)\right), \\
i=1, \ldots, n-1, j=1, \ldots, s .
\end{gathered}
$$

The superscript $T$ denotes transpose. The component that is in $r$ th row, $k$ th column, $1 \leq r, k \leq s$ of matrix $A_{p}, 0 \leq p \leq$ $n$ will be denoted by $a_{r, k}^{p}$. Then, (8) can be replaced by the following equivalent system in parametric form:

$$
\begin{aligned}
& \frac{\sum_{i=0}^{n} \sum_{k=1}^{s} a_{d, k}^{i}(t) y_{k}^{(i)}(t, r)}{\overline{\sum_{i=0}^{n} \sum_{k=1}^{s} a_{d, k}^{i}(t) y_{k}^{(i)}(t, r)}}=\overline{f_{d}(t, r),} \quad d=1, \ldots, s, \\
& \underline{y_{d}^{(i)}(t, r)}, \quad d=1, \ldots, s, \\
& \overline{y_{j}^{(i)}}(0, r)=\underline{g_{i j}}(r), \quad i=0, \ldots, n-1, j=1, \ldots, s, \\
& \overline{g_{i j}}(r), \quad i=0, \ldots, n-1, \quad j=1, \ldots, s,
\end{aligned}
$$

where $r \in[0,1]$.

We define functions $b_{d, k}^{i}(t)$ and $c_{d, k}^{i}(t) ; 1 \leq d, k \leq s, 0 \leq$ $i \leq n$, in the following form:

$$
\begin{aligned}
& b_{d, k}^{i}(t)= \begin{cases}0, & a_{d, k}^{i}(t)<0, \\
a_{d, k}^{i}(t), & a_{d, k}^{i}(t) \geq 0,\end{cases} \\
& c_{d, k}^{i}(t)= \begin{cases}a_{d, k}^{i}(t), & a_{d, k}^{i}(t)<0, \\
0, & a_{d, k}^{i}(t) \geq 0 .\end{cases}
\end{aligned}
$$


Now (11) becomes as follows:

$$
\begin{aligned}
\sum_{i=0}^{n} \sum_{k=1}^{s}\left[b_{d, k}^{i}(t)\left(y_{l}\right)_{k}^{(i)}(t, r)+c_{d, k}^{i}(t)\left(y_{u}\right)_{k}^{(i)}(t, r)\right] & =\left(f_{l}\right)_{d}(t, r), \\
d & =1, \ldots, s \\
\sum_{i=0}^{n} \sum_{k=1}^{s}\left[c_{d, k}^{i}(t)\left(y_{l}\right)_{k}^{(i)}(t, r)+b_{d, k}^{i}(t)\left(y_{u}\right)_{k}^{(i)}(t, r)\right] & =\left(f_{u}\right)_{d}(t, r), \\
d & =1, \ldots, s \\
\left(y_{l}\right)_{j}^{(i)}(0, r)=\left(g_{l}\right)_{i j}(r), \quad i=0, \ldots, n-1, j & =1, \ldots, s, \\
\left(y_{u}\right)_{j}^{(i)}(0, r)=\left(g_{u}\right)_{i j}(r), \quad i=0, \ldots, n-1, j & =1, \ldots, s .
\end{aligned}
$$

\section{Basic Ideas of HAM}

We consider the following differential equations:

$$
\sum_{i=0}^{n} A_{i}(t) Z^{(i)}(t, r)-F(t, r)=0,
$$

where

$$
\begin{aligned}
& Z(t, r)=\left[z_{1}(t, r), z_{2}(t, r), \ldots, z_{s}(t, r)\right]^{T}, \\
& F(t, r)=\left[f_{1}(t, r), f_{2}(t, r), \ldots, f_{s}(t, r)\right]^{T},
\end{aligned}
$$

and $A_{0}(t), A_{1}(t), \ldots, A_{n-1}(t)$ are $s \times s$ matrixes and every component of them is a real function of $t$ and $A_{n}(t)=I_{s}$, where $I_{s}$ denotes the $s \times s$ identity matrix.

The above system of equations can be written in following form:

$$
\mathcal{N}_{i}\left[z_{1}(t, r), z_{2}(t, r), \ldots, z_{s}(t, r)\right]=0, \quad i=1,2, \ldots, s,
$$

where $\mathcal{N}_{i}$ are nonlinear operators that represent the whole equations, $t$ and $r$ denote the independent variables, and $z_{i}(t, r), i=1,2, \ldots, s$ are unknown functions, respectively. By means of generalizing the traditional homotopy method constructed the so-called zero-order deformation equations

$$
\begin{array}{r}
(1-q) \mathcal{L}_{i}\left[\phi_{i}(t, r ; q)-z_{i, 0}(t, r)\right] \\
=q \hbar \mathcal{N}_{i}\left[\phi_{1}(t, r ; q), \ldots, \phi_{n}(t, r ; q)\right], \\
i=1, \ldots, n,
\end{array}
$$

where $q \in[0,1]$ is an embedding parameter, $\hbar$ is nonzero auxiliary parameter, $\mathcal{L}_{i}, i=1,2, \ldots, s$ are auxiliary linear operators, $z_{i, 0}(t, r)$ are initial guesses of $z_{i}(t, r)$, and $\phi_{i}(t, r ; q)$ are unknown functions. It is important to note that one has great freedom to choose auxiliary objects such as $\hbar$ and $\mathcal{L}_{i}$ in HAM. Obviously, when $q=0$ and $q=1$, both

$$
\phi_{i}(t, r ; 0)=z_{i, 0}(t, r), \quad \phi_{i}(t, r ; 1)=z_{i}(t, r)
$$

hold. Thus, as $q$ increases from 0 to 1 , the solutions $\phi_{i}(t, r ; q)$ varies from the initial guesses $z_{i, 0}(t, r)$ to the solutions $z_{i}(t, r)$.
Expanding $\phi_{i}(t, r ; q)$ in Taylor's series with respect to $q$, one has

$$
\phi_{i}(t, r ; q)=z_{i, 0}(t, r)+\sum_{m=1}^{+\infty} z_{i, m}(t, r) q^{m}
$$

where

$$
z_{i, m}=\left.\frac{1}{m !} \frac{\partial^{m} \phi_{i}(t, r ; q)}{\partial q^{m}}\right|_{q=0}
$$

If auxiliary linear operator, initial guesses, and auxiliary parameter $\hbar$ are properly chosen, then the series equations (19) converges at $q=1$ and

$$
\begin{array}{r}
\phi_{i}(t, r ; 1)=z_{i, 0}(t, r)+\sum_{m=1}^{+\infty} z_{i, m}(t, r), \\
i=1, \ldots, s,
\end{array}
$$

which must be solution of the original nonlinear equations.

According to (20), the governing equations can be deduced from the zero-order deformation equations (17). Define the vectors

$$
\vec{z}_{i, m}=\left\{z_{i, 0}(t, r), z_{i, 1}(t, r), \ldots, z_{i, m}(t, r)\right\}, \quad i=1, \ldots, s .
$$

Differentiating (17) $m$ time with respect to the embedding parameter $q$ and then setting $q=0$ and finally dividing them by $m$ !, we have the so-called $m$ th order deformation equations

$$
\begin{array}{r}
\mathcal{L}_{i}\left[z_{i, m}(t, r)-\chi_{m} z_{i, m-1}(t, r)\right] \\
=\hbar \mathcal{R}_{i, m}\left(\vec{z}_{1, m-1}, \ldots, \vec{z}_{s, m-1}\right) \\
i=1, \ldots, s,
\end{array}
$$

where

$$
\begin{gathered}
\mathcal{R}_{i, m}\left(\vec{z}_{1, m-1}, \ldots, \vec{z}_{s, m-1}\right) \\
=\left.\frac{1}{(m-1) !} \frac{\partial^{m-1} \mathcal{N}_{i}\left[\phi_{1}(t, r ; q), \ldots, \phi_{s}(t, r ; q)\right]}{\partial q^{m-1}}\right|_{q=0}, \\
i=1, \ldots, s, \\
\chi_{m}= \begin{cases}0, & m \leq 1, \\
1, & m>1 .\end{cases}
\end{gathered}
$$

Now, to simplify and solve (11), we need to know the sign of components of $A_{i}(t) ; 0 \leq i \leq n$ and use of Remark 2. Also we should be noted that in case that some components of $A_{0}(t), A_{1}(t), \ldots, A_{n}(t)$ for some $t$ are negative, we must divide the defined interval for $t$ into small intervals, so that sign of each component of $A_{0}(t), A_{1}(t), \ldots, A_{n}(t)$ in each small interval be unchanged. Now for each small interval we have a separate system $2 s \times s(n+1)$. 


\section{Convergency of HAM for System of Fuzzy Differential Equations}

In this section, we prove a theorem which shows the convergency of approximate HAM solution applied for (8).
First we define $\mathcal{R}_{k, m}\left[\left(\vec{y}_{l}\right)_{1, m-1}, \ldots,\left(\vec{y}_{l}\right)_{s, m-1},\left(\vec{y}_{u}\right)_{1, m-1}\right.$, $\left.\ldots,\left(\vec{y}_{u}\right)_{s, m-1}\right] ; 1 \leq k \leq s$, in the following form:

$$
\mathcal{R}_{k, m}=\frac{1}{(m-1) !} \frac{\partial^{m-1} \mathcal{N}_{k}\left[\left(y_{l}\right)_{1, m-1}, \ldots,\left(y_{l}\right)_{s, m-1},\left(y_{u}\right)_{1, m-1}, \ldots,\left(y_{u}\right)_{s, m-1}\right]}{\partial q^{m-1}} \mid q=0
$$

if $k$ th equation of (13) is inclusive $b_{d, k}^{i}(t)\left(y_{l}\right)_{k}^{(i)}(t, r) ; 1 \leq i \leq$

$n, 1 \leq d \leq s$, and otherwise

$$
\mathcal{R}_{k, m}=\frac{1}{(m-1) !} \frac{\partial^{m-1} \mathcal{N}_{k+s}\left[\left(y_{l}\right)_{1, m-1}, \ldots,\left(y_{l}\right)_{s, m-1},\left(y_{u}\right)_{1, m-1}, \ldots,\left(y_{u}\right)_{s, m-1}\right]}{\partial q^{m-1}} \mid q=0 .
$$

Theorem 6. Let the series $\sum_{m=1}^{\infty}(y)_{k, m}(t, r) ; 1 \leq k \leq s$ be uniformly converge to $(y)_{k}(t, r) ; 1 \leq k \leq s$. Then $\left(\left(y_{l}\right)_{k}(t, r),\left(y_{u}\right)_{k}(t, r)\right), 1 \leq k \leq s$ is exact solution of (13).

Proof. Since $\sum_{m=1}^{\infty}(y)_{k, m}(t, r), 1 \leq k \leq s$ is convergent, we must have

$$
\lim _{m \rightarrow \infty}(y)_{k, m}(t, r)=0, \quad 1 \leq k \leq s, 0 \leq t \leq T, 0 \leq r \leq 1 .
$$

On the other hand, since $\mathcal{L}_{i}$ are linear operators, thus

$$
\begin{aligned}
\sum_{m=1}^{n} \mathcal{L}_{k}\left[(y)_{k, m}(t, r)-\chi_{m}(y)_{k, m-1}(t, r)\right] \\
=\sum_{m=1}^{n}\left[\mathcal{L}_{k}(y)_{k, m}(t, r)-\chi_{m} \mathcal{L}_{k}\left[(y)_{k, m-1}(t, r)\right]\right] \\
=\mathcal{L}_{k}\left[(y)_{k, 1}(t, r)\right]+\left(\mathcal{L}_{k}\left[(y)_{k, 2}(t, r)\right]\right. \\
\left.\quad-\mathcal{L}_{k}\left[(y)_{k, 1}(t, r)\right]\right) \\
+\cdots+\left(\mathcal{L}_{k}\left[(y)_{k, n}(t, r)\right]-\mathcal{L}_{k}\left[(y)_{k, n-1}(t, r)\right]\right) \\
=\mathcal{L}_{k}\left[(y)_{k, n}(t, r)\right], \quad k=1,2, \ldots, s,
\end{aligned}
$$

$$
\begin{aligned}
& \sum_{m=1}^{\infty} \mathcal{R}_{k, m}\left[\left(\vec{y}_{l}\right)_{1, m-1}, \ldots,\left(\overrightarrow{y_{l}}\right)_{s, m-1},\left(\overrightarrow{y_{u}}\right)_{1, m-1}, \ldots,\left(\overrightarrow{y_{u}}\right)_{s, m-1}\right] \\
& \quad=0 .
\end{aligned}
$$$$
\left.\left(\overrightarrow{y_{u}}\right)_{1, m-1}, \ldots,\left(\overrightarrow{y_{u}}\right)_{s, m-1}\right]
$$$$
=\sum_{m=1}^{\infty} \mathcal{L}_{k}\left[(y)_{k, m}(t, r)-\chi_{m}(y)_{k, m-1}(t, r)\right]=0,
$$$$
k=1,2, \ldots, s
$$

Since $\hbar \neq 0$, thus from the above equations for $k=1,2, \ldots, s$, we can write

From uniform convergency we have

$$
\begin{array}{r}
\sum_{m=1}^{\infty} \mathcal{L}_{k}\left[(y)_{k, m}(t, r)-\chi_{m}(y)_{k, m-1}(t, r)\right] \\
=\lim _{n \rightarrow \infty} \mathcal{L}_{k}\left[(y)_{k, n}(t, r)\right]=\mathcal{L}_{k}\left[\lim _{n \rightarrow \infty}(y)_{k, n}(t, r)\right]=0, \\
k=1,2, \ldots, s,
\end{array}
$$$$
\begin{array}{r}
\sum_{m=1}^{\infty} \frac{\partial^{i}}{\partial t^{i}}(y)_{k, m-1}(t, r)=\frac{\partial^{i}}{\partial t^{i}}(y)_{k}(t, r), \quad i=0,1, \ldots, n, \\
k=1,2, \ldots, s,
\end{array}
$$ 
thus

$$
\begin{aligned}
0 & =\sum_{m=1}^{\infty}\left[\sum_{i=0}^{n} A_{i}(t) Y^{(i)}(t)-\left(1-\chi_{m}\right)(F)_{d}(t, r)\right] \\
& =\sum_{i=0}^{n} A_{i}(t) \sum_{m=1}^{\infty} Y^{(i)}(t)-\sum_{m=1}^{\infty}\left(1-\chi_{m}\right)(F)_{d}(t, r) \\
& =\sum_{i=0}^{n} A_{i}(t) \sum_{m=1}^{\infty} \frac{\partial^{i}}{\partial t^{i}}(Y)_{k, m-1}(t, r)-(F)_{d}(t, r) \\
& =\sum_{i=0}^{n} A_{i}(t) \frac{\partial^{i}}{\partial t^{i}}(Y)_{k}(t, r)-(F)_{d}(t, r), \quad d=1, \ldots, s .
\end{aligned}
$$

Therefore, $\left\{y_{k}(t, r)=\left(\left(y_{l}\right)_{k}(t, r),\left(y_{u}\right)_{k}(t, r)\right) ; k=1,2, \ldots s\right\}$ is the exact solution of (11) and $Y=\left[y_{1}(t, r), y_{2}(t, r)\right.$, $\left.\ldots, y_{s}(t, r)\right]^{T}$ is the exact solution of (8) and proof is completed.

\section{Illustrative Examples}

Example 7. Consider the following second-order fuzzy linear differential equation:

$$
\begin{gathered}
Y^{\prime \prime}+Y=-t, \quad t \in[0,1] \\
Y(0)=(0.1 r-0.1,0.1-0.1 r), \\
Y^{\prime}(0)=(0.088+0.1 r, 0.288-0.1 r) .
\end{gathered}
$$

The exact solution is as follows:

$$
\begin{aligned}
& \underline{Y}(t, r)=(0.1 r-0.1) \cos (t)+(1.088+0.1 r) \sin (t)-t, \\
& \bar{Y}(t, r)=(0.1-0.1 r) \cos (t)+(1.288-0.1 r) \sin (t)-t .
\end{aligned}
$$

According to (11), we may replace (34) by the following equivalent system:

$$
\begin{gathered}
\underline{Y}^{\prime \prime}+\underline{Y}=-t, \quad t \in[0,1], \\
\bar{Y}^{\prime \prime}+\bar{Y}=t, \quad t \in[0,1], \\
\underline{Y}(0)=0.1 r-0.1, \quad \underline{Y}^{\prime}(0)=0.088+0.1 r, \\
\bar{Y}(0)=0.1-0.1 r, \quad \bar{Y}^{\prime}(0)=0.288-0.1 r .
\end{gathered}
$$

We first construct the zero-order deformation equations

$$
\begin{array}{r}
(1-q) \mathcal{L}_{i}\left[\phi_{i}(t, r ; q)-z_{i, 0}(t, r)\right]=q \hbar \mathcal{N}_{i}\left[\phi_{i}(t, r ; q)\right], \\
i=1,2,
\end{array}
$$

subject to the initial conditions

$$
\begin{gathered}
\underline{Y}_{0}=(0.1 r-0.1)+(0.088+0.1 r) t, \\
\overline{Y_{0}}=(0.1-0.1 r)+(0.288-0.1 r) t,
\end{gathered}
$$

and the linear operator

$$
\mathcal{L}_{i}\left[\phi_{i}(t, r ; q)\right]=\frac{\sigma^{2} \phi_{i}(t, r ; q)}{\sigma t^{2}}, \quad i=1,2,
$$

with the property

$$
\mathcal{L}_{i}\left[c_{i 0}+t c_{i 1}\right]=0, \quad i=1,2,
$$

where $c_{i 0}, c_{i 1}(i=1,2)$ are integral constants. Also from (36), we can define

$$
\begin{aligned}
& \mathcal{N}_{1}\left[\phi_{1}(t, r ; q)\right]=\frac{\sigma^{2} \phi_{1}(t, r ; q)}{\sigma t^{2}}+\phi_{1}(t, r ; q)+t, \\
& \mathcal{N}_{2}\left[\phi_{2}(t, r ; q)\right]=\frac{\sigma^{2} \phi_{2}(t, r ; q)}{\sigma t^{2}}+\phi_{2}(t, r ; q)+t .
\end{aligned}
$$

Obviously, when $q=0$ and $q=1$,

$$
\begin{array}{ll}
\phi_{1}(t, r ; 0)=z_{1,0}(t, r)=\underline{Y}_{0}, & \phi_{1}(t, r ; 1)=\underline{Y}, \\
\phi_{2}(t, r ; 0)=z_{2,0}(t, r)=\bar{Y}_{0}, & \phi_{2}(t, r ; 1)=\bar{Y} .
\end{array}
$$

Therefore, when the embedding parameter $q$ increases from 0 to 1 , the homotopy solutions $\phi_{i}(t, r, q)$ vary from $z_{i, 0}(t, r)$ to the solutions $z_{i}(t, r)$ for $i=1,2$. Now, by expanding $\phi_{i}(t, r ; q)$ in Taylor's series with respect to $q$, we have

$$
\phi_{i}(t, r ; q)=z_{i, 0}(t, r)+\sum_{m=1}^{+\infty} z_{i, m}(t, r) q^{m}, \quad i=1,2,
$$

where

$$
z_{i, m}(t, r)=\left.\frac{1}{m !} \frac{\partial^{m} \phi_{i}(t, r ; q)}{\partial q^{m}}\right|_{q=0}, \quad i=1,2 .
$$

Assuming that auxiliary parameter $\hbar$, the initial guesses and the auxiliary linear operator are properly chosen, then the above series is convergent at $q=1$, and

$$
\begin{aligned}
& \underline{Y}(t, r)=z_{1,0}(t, r)+\sum_{m=1}^{\infty} z_{1, m}(t, r), \\
& \bar{Y}(t, r)=z_{2,0}(t, r)+\sum_{m=1}^{\infty} z_{2, m}(t, r) .
\end{aligned}
$$

The $m$ th order deformation equations are

$$
\begin{array}{r}
\mathcal{L}_{i}\left[z_{i, m}(t, r)-\chi_{m} z_{i, m-1}(t, r)\right]=\hbar \mathcal{R}_{i, m}\left(\vec{z}_{i, m-1}\right), \\
i=1,2,
\end{array}
$$

with the initial conditions

$$
z_{i, m}(0, r)=0, \quad i=1,2,
$$

where

$$
\mathcal{R}_{i, m}\left(\vec{z}_{i, m-1}\right)=\frac{\partial^{2} z_{i, m-1}}{\partial t^{2}}+z_{i, m-1}+\left(1-\chi_{m}\right) t, \quad i=1,2 .
$$


Therefore, we recursively obtain

$$
\begin{aligned}
z_{1,1}(t, r)= & \frac{-\hbar t^{2}}{20}+\frac{\hbar r t^{2}}{20}+\frac{68 \hbar t^{3}}{375}+\frac{\hbar r t^{3}}{60}, \\
z_{1,2}(t, r)= & \frac{-\hbar t^{2}}{20}-\frac{-\hbar^{2} t^{2}}{20}+\frac{\hbar r t^{2}}{20} \\
& +\frac{\hbar^{2} r t^{2}}{20}+\frac{68 \hbar t^{3}}{375}+\frac{68 \hbar^{2} t^{3}}{375} \\
& +\frac{\hbar r t^{3}}{60}+\frac{\hbar^{2} r t^{3}}{60}-\frac{\hbar^{2} t^{4}}{240} \\
& +\frac{\hbar^{2} r t^{4}}{240}+\frac{17 \hbar^{2} t^{5}}{1875}+\frac{\hbar^{2} r t^{5}}{1200}, \\
z_{2,1}(t, r)=\frac{\hbar t^{2}}{20} & -\frac{\hbar r t^{2}}{20}+\frac{161 \hbar t^{3}}{750}-\frac{\hbar r t^{3}}{60}, \\
z_{2,2}(t, r)= & \frac{\hbar t^{2}}{20}+\frac{-\hbar^{2} t^{2}}{20}-\frac{\hbar r t^{2}}{20} \\
& -\frac{\hbar^{2} r t^{2}}{20}+\frac{161 \hbar t^{3}}{750}+\frac{161 \hbar^{2} t^{3}}{750} \\
& -\frac{\hbar r t^{3}}{60}-\frac{\hbar^{2} r t^{3}}{60}+\frac{\hbar^{2} t^{4}}{240} \\
\vdots & -\frac{\hbar^{2} r t^{4}}{240}+\frac{161 \hbar^{2} t^{5}}{15000}-\frac{\hbar^{2} r t^{5}}{1200}, \\
\vdots &
\end{aligned}
$$

Then the solutions obtained by HAM are as follows:

$$
\begin{aligned}
\underline{Y}(t, r)= & z_{1,0}(t, r)+z_{1,1}(t, r) \\
& +z_{1,2}(t, r)+z_{1,3}(t, r)+\cdots, \\
\bar{Y}(t, r)= & z_{2,0}(t, r)+z_{2,1}(t, r) \\
& +z_{2,2}(t, r)+z_{2,3}(t, r)+\cdots .
\end{aligned}
$$

Figures 1 and 3 show the $\hbar$-mesh of $\underline{Y}$ and $\bar{Y}$ to get a proper interval for convergency. $\hbar$-mesh is a generalization of traditional Contour plots, and for connection between $\hbar$-meshes and Contour plots we plot Figures 2 and 4 . Also to find the best quantity of $\hbar$ that lies in the convergency interval, we use the residual error of norm 2 as follows:

$$
\operatorname{Res}\left[\underline{Y}_{n}\right]=\left[\iint_{0}^{1}\left(\underline{Y}_{n}(t, r)-\underline{Y}(t, r)\right)^{2} d t d r\right]^{1 / 2},
$$

which is a function with respect to $\hbar$. Now, by minimizing the $\operatorname{Res}\left[\underline{Y}_{10}\right]$ we obtain the best choice for auxiliary parameter to approximate of $\underline{Y}_{10}(t, r)$ as follows:

$$
\hbar=-1.02698
$$

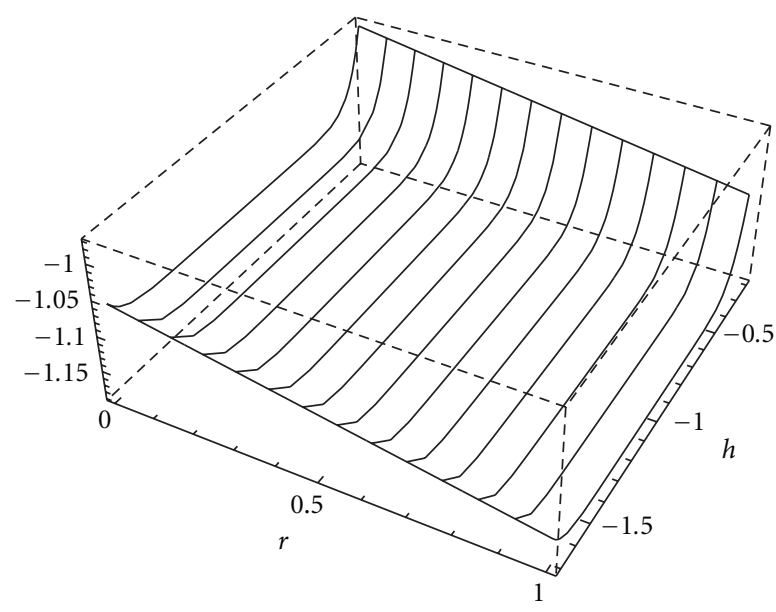

Figure 1: $\hbar$-mesh of $\left(\partial^{3} / \partial t^{3}\right) \underline{Y}_{10}(0, r)$.

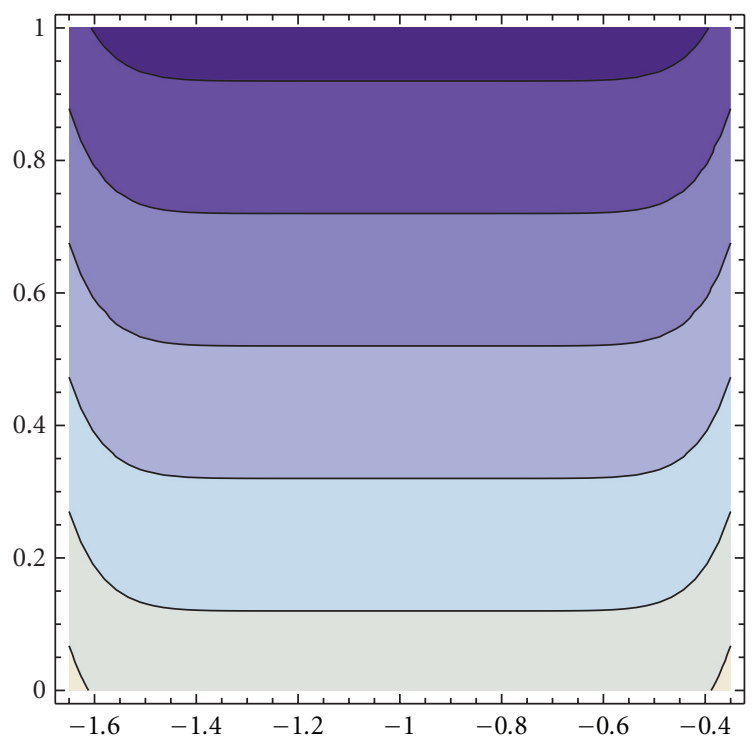

Figure 2: Contour plot of $\left(\partial^{3} / \partial t^{3}\right) \underline{Y}_{10}(0, r)$.



FIGURE 3: $\hbar$-mesh of $\left(\partial^{3} / \partial t^{3}\right) \bar{Y}_{10}(0, r)$. 


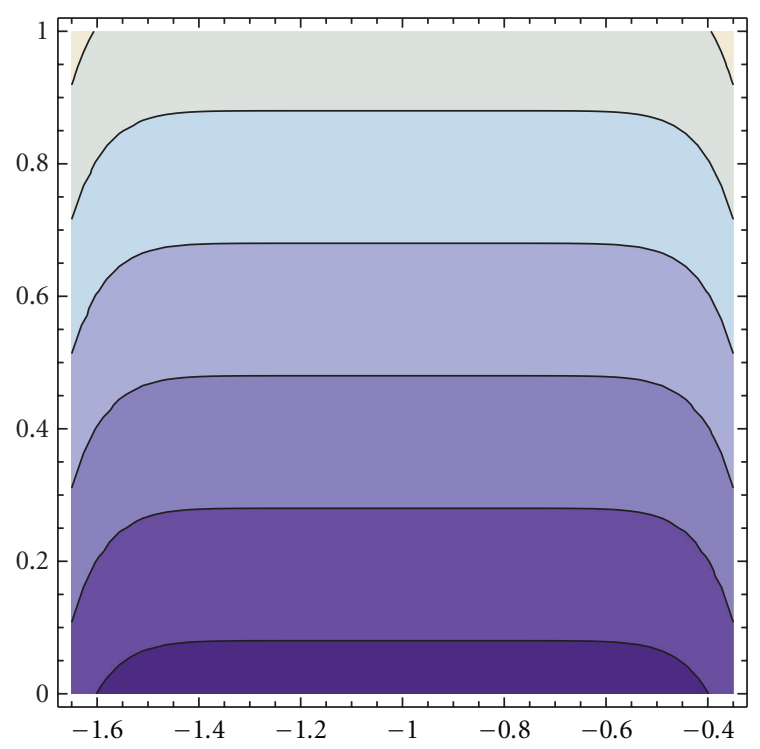

Figure 4: Contour plot of $\left(\partial^{3} / \partial t^{3}\right) \bar{Y}_{10}(0, r)$.

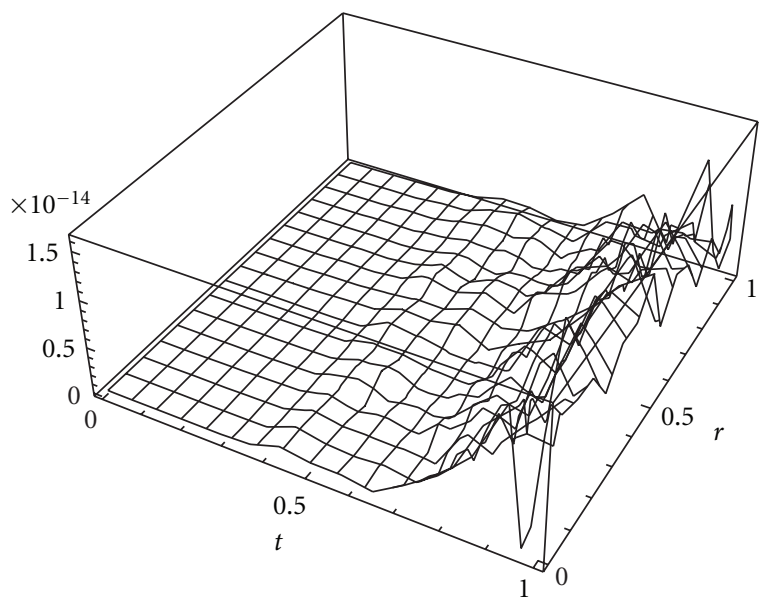

Figure 5: Absolute error of $\underline{Y}_{10}(t, r)$ for $\hbar=-1.02698$.

and in this case absolute error for the 10th order approximation by HAM for $\underline{Y}_{n}(t, r)$ is plotted in Figure 5 .

By minimizing the residual error defined by

$$
\operatorname{Res}\left[\bar{Y}_{n}\right]=\left[\iint_{0}^{1}\left(\bar{Y}_{n}(t, r)-\bar{Y}(t, r)\right)^{2} d t d r\right]^{1 / 2},
$$

it is clear that the best choice for auxiliary parameter to approximate $\bar{Y}_{10}(t, r)$ is

$$
\hbar=-1.02683
$$

which in this case absolute error for the 10th order approximation by HAM for $\bar{Y}_{n}(t, r)$ is plotted in Figure 6.

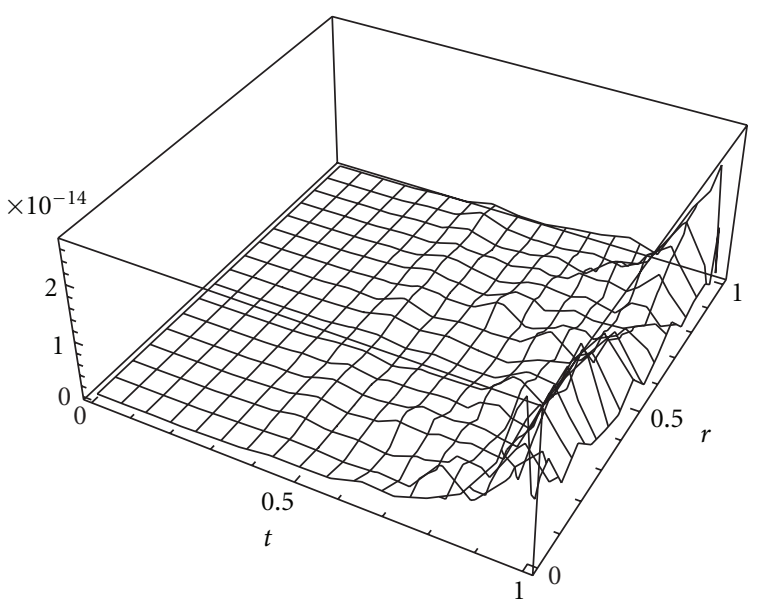

Figure 6: Absolute error of $\bar{Y}_{10}(t, r)$ for $\hbar=-1.02683$.

Example 8. Consider the system of fuzzy differential equation

$$
\begin{aligned}
Y^{\prime \prime}+e^{2 t} G^{\prime}= & \left(\left(4 r^{2}+5 r-1\right) e^{2 t},\right. \\
& \left.\left(-4 r^{3}-2 r t+2 t+12\right) e^{2 t}\right), \\
Y^{\prime}+G^{\prime \prime}= & \left(\left(2 r^{2}+2 r\right) e^{2 t},\right. \\
& \left.\left(-2 r^{3}+6\right) e^{2 t}-2 r+2\right), \\
Y(0)= & \left(r^{2}+r, 3-r^{3}\right), \\
Y^{\prime}(0)= & \left(2 r^{2}+2 r,-2 r^{3}+6\right), \\
G(0)= & (0,0), \quad G^{\prime}(0)=(r-1,0), \quad t \in[0,1] .
\end{aligned}
$$

The exact solution is as follows:

$$
\begin{aligned}
& Y(t, r)=\left(\left(r^{2}+r\right) e^{2 t},\left(3-r^{3}\right) e^{2 t}\right), \\
& G(t, r)=\left((r-1) t,(1-r) t^{2}\right) .
\end{aligned}
$$

We may replace (55) by the equivalent system

$$
\begin{gathered}
\underline{Y}^{\prime \prime}+e^{2 t} \underline{G}^{\prime}=\left(4 r^{2}+5 r-1\right) e^{2 t}, \\
\bar{Y}^{\prime \prime}+e^{2 t} \bar{G}^{\prime}=\left(-4 r^{3}-2 r t+2 t+12\right) e^{2 t}, \\
\underline{Y}^{\prime}+\underline{G}^{\prime \prime}=\left(2 r^{2}+2 r\right) e^{2 t}, \\
\bar{Y}^{\prime}+\bar{G}^{\prime \prime}=\left(-2 r^{3}+6\right) e^{2 t}-2 r+2, \\
\underline{Y}(0, r)=r^{2}+r, \quad \bar{Y}(0, r)=3-r^{3}, \\
\underline{Y^{\prime}}(0, r)=2 r^{2}+2 r, \quad \bar{Y}^{\prime}(0, r)=-2 r^{3}+6, \\
\underline{G}(0, r)=0, \quad \bar{G}(0, r)=0, \\
\underline{G}^{\prime}(0, r)=r-1, \quad \bar{G}^{\prime}(0, r)=0 .
\end{gathered}
$$

We select the following initial guesses:

$$
\begin{array}{cc}
\underline{Y}_{0}=r^{2}+r+\left(2 r^{2}+2 r\right) t, & \bar{Y}_{0}=3-r^{3}+\left(-2 r^{3}+6\right) t, \\
\underline{G}_{0}=(r-1) t, & \bar{G}_{0}=2-r,
\end{array}
$$


and the auxiliary linear operators

$$
\mathcal{L}_{i}\left[\phi_{i}(t, r ; q)\right]=\frac{\partial^{2} \phi_{i}(t, r ; q)}{\partial t^{2}}, \quad i=1,2,3,4,
$$

with the property

$$
\mathcal{L}_{i}\left[c_{i 0}+t c_{i 1}\right]=0, \quad i=1,2,3,4,
$$

where $c_{i 0}$ and $c_{i 1}(i=1,2,3,4)$ are integral constants. Also the nonlinear operators are

$$
\begin{aligned}
& \mathcal{N}_{1}\left[\phi_{1}(t, r ; q), \ldots, \phi_{4}(t, r ; q)\right] \\
& =\frac{\partial^{2} \phi_{1}(t, r ; q)}{\partial t^{2}}+e^{2 t} \frac{\partial \phi_{3}(t, r ; q)}{\partial t}-\left(4 r^{2}+5 r-1\right) e^{2 t}, \\
& \mathcal{N}_{2}\left[\phi_{1}(t, r ; q), \ldots, \phi_{4}(t, r ; q)\right] \\
& =\frac{\partial^{2} \phi_{2}(t, r ; q)}{\partial t^{2}}-\frac{\partial \phi_{4}(t, r ; q)}{\partial t}-\left(-4 r^{3}-2 r t+2 t+12\right) e^{2 t}, \\
& \mathcal{N}_{3}\left[\phi_{1}(t, r ; q), \ldots, \phi_{4}(t, r ; q)\right] \\
& =\frac{\partial^{2} \phi_{3}(t, r ; q)}{\partial t^{2}}+\frac{\partial \phi_{1}(t, r ; q)}{\partial t}-\left(2 r^{2}+2 r\right) e^{2 t}, \\
& \mathcal{N}_{4}\left[\phi_{1}(t, r ; q), \ldots, \phi_{4}(t, r ; q)\right] \\
& =\frac{\partial^{2} \phi_{4}(t, r ; q)}{\partial t^{2}}+\frac{\partial \phi_{2}(t, r ; q)}{\partial t}-\left(\left(-2 r^{3}+6\right) e^{2 t}-2 r+2\right) .
\end{aligned}
$$

Then $m$ th order deformation equations are

$$
\begin{array}{r}
\mathcal{L}_{i}\left[z_{i, m}(t, r)-\chi_{m} z_{i, m-1}(t, r)\right]=\hbar \mathcal{R}_{i, m}\left(\vec{z}_{1, m-1}, \ldots, \vec{z}_{4, m-1}\right), \\
i=1,2,3,4,
\end{array}
$$

with the initial conditions

$$
\begin{gathered}
z_{i, m}(0, r)=0, \quad i=1,2,3,4, \\
\mathcal{R}_{1, m}\left(\vec{z}_{1, m-1}, \ldots, \vec{z}_{4, m-1}\right) \\
=\frac{\partial^{2} z_{1, m-1}}{\partial t^{2}}+e^{2 t} \frac{\partial z_{3, m-1}}{\partial t} \\
-\left(1-\chi_{m}\right)\left(4 r^{2}+5 r-1\right) e^{2 t}, \\
\mathcal{R}_{2, m}\left(\vec{z}_{1, m-1}, \ldots, \vec{z}_{4, m-1}\right) \\
=\frac{\partial^{2} z_{2, m-1}}{\partial t^{2}}-\frac{\partial z_{4, m-1}}{\partial t} \\
-\left(1-\chi_{m}\right)\left(-4 r^{3}-2 r t+2 t+12\right) e^{2 t}, \\
\mathcal{R}_{3, m}\left(\vec{z}_{1, m-1}, \ldots, \vec{z}_{4, m-1}\right) \\
=\frac{\partial^{2} z_{3, m-1}}{\partial t^{2}}+\frac{\partial z_{1, m-1}}{\partial t} \\
-\left(1-\chi_{m}\right)\left(2 r^{2}+2 r\right) e^{2 t}, \\
\mathcal{R}_{4, m}\left(\vec{z}_{1, m-1}, \ldots, \vec{z}_{4, m-1}\right) \\
=\frac{\partial^{2} z_{4, m-1}}{\partial t^{2}}+\frac{\partial z_{2, m-1}}{\partial t} \\
-\left(1-\chi_{m}\right)\left(\left(-2 r^{3}+6\right) e^{2 t}-2 r+2\right) .
\end{gathered}
$$

Therefore, we recursively obtain

$$
\begin{aligned}
z_{1,1}(t, r)= & \hbar r-e^{2 t} \hbar r+\hbar r^{2}-e^{2 t} \hbar r^{2}+2 \hbar r t+2 \hbar r^{2} t, \\
z_{1,2}(t, r)= & \hbar r-e^{2 t} \hbar r+\frac{21 \hbar^{2} r}{16}-\frac{5}{4} e^{2 t} \hbar^{2} r-\frac{1}{16} e^{4 t} \hbar^{2} r \\
& +\hbar r^{2}-e^{2 t} \hbar r^{2}+\frac{21 \hbar^{2} r^{2}}{16} \\
& -\frac{5}{4} e^{2 t} \hbar^{2} r^{2}-\frac{1}{16} e^{4 t} \hbar^{2} r^{2}+2 \hbar r t \\
& +\frac{9}{4} \hbar^{2} r t+\frac{1}{2} e^{2 t} \hbar^{2} r t+2 \hbar r^{2} t \\
+ & \frac{9}{4} \hbar^{2} r^{2} t+\frac{1}{2} e^{2 t} \hbar^{2} r^{2} t, \\
z_{2,1}(t, r) & =\frac{5 \hbar}{2}-\frac{5}{2} e^{2 t} \hbar+\frac{\hbar r}{2}-\frac{1}{2} e^{2 t} \hbar r-\hbar r^{3} \\
& +e^{2 t} \hbar r^{3}+\frac{11 \hbar t}{2}-\frac{1}{2} e^{2 t} \hbar t \\
& +\frac{\hbar r t}{2}+\frac{1}{2} e^{2 t} \hbar r t-2 \hbar r^{3} t,
\end{aligned}
$$

$z_{2,2}(t, r)=\frac{5 \hbar}{2}-\frac{5}{2} e^{2 t} \hbar$

$$
\begin{aligned}
& +\frac{47 \hbar^{2}}{16}-\frac{11}{4} e^{2 t} \hbar^{2}-\frac{3}{16} e^{4 t} \hbar^{2}+\frac{\hbar r}{2} \\
& -\frac{1}{2} e^{2 t} \hbar r+\hbar^{2} r-e^{2 t} \hbar^{2} r-\hbar r^{3} \\
& +e^{2 t} \hbar r^{3}-\frac{21 \hbar^{2} r^{3}}{16}+\frac{5}{4} e^{2 t} \hbar^{2} r^{3}+\frac{1}{16} e^{4 t} \hbar^{2} r^{3} \\
& +\frac{11 \hbar t}{2}-\frac{1}{2} e^{2 t} \hbar t+\frac{23 \hbar^{2} t}{4}+\frac{1}{2} e^{2 t} \hbar^{2} t \\
& +\frac{\hbar r t}{2}+\frac{1}{2} e^{2 t} \hbar r t+\hbar^{2} r t+e^{2 t} \hbar^{2} r t \\
& -2 \hbar r^{3} t-\frac{9}{4} \hbar^{2} r^{3} t-\frac{1}{2} e^{2 t} \hbar^{2} r^{3} t, \\
& z_{3,1}(t, r)=\frac{\hbar r}{2}-\frac{1}{2} e^{2 t} \hbar r+\frac{\hbar r^{2}}{2}-\frac{1}{2} e^{2 t} \hbar r^{2}+\hbar r t \\
& +\hbar r^{2} t+\hbar r t^{2}+\hbar r^{2} t^{2} \\
& z_{3,2}(t, r)=\frac{\hbar r}{2}-\frac{1}{2} e^{2 t} \hbar r \\
& +\hbar^{2} r-e^{2 t} \hbar^{2} r+\frac{\hbar r^{2}}{2}-\frac{1}{2} e^{2 t} \hbar r^{2} \\
& +\hbar^{2} r^{2}-e^{2 t} \hbar^{2} r^{2}+\hbar r t+2 \hbar^{2} r t \\
& +\hbar r^{2} t+2 \hbar^{2} r^{2} t+\hbar r t^{2} \\
& +2 \hbar^{2} r t^{2}+\hbar r^{2} t^{2}+2 \hbar^{2} r^{2} t^{2}, \\
& z_{4,1}(t, r)=\frac{3 \hbar}{2}-\frac{3}{2} e^{2 t} \hbar-\frac{\hbar r^{3}}{2}+\frac{1}{2} e^{2 t} \hbar r^{3}+3 \hbar t-\hbar r^{3} t \\
& +2 \hbar t^{2}+\hbar r t^{2}-\hbar r^{3} t^{2},
\end{aligned}
$$




$$
\begin{aligned}
z_{4,2}(t, r)= & \frac{3 \hbar}{2}-\frac{3}{2} e^{2 t} \hbar+\frac{21 \hbar^{2}}{8} \\
& -\frac{21}{8} e^{2 t} \hbar^{2}+\frac{3 \hbar^{2} r}{8}-\frac{3}{8} e^{2 t} \hbar^{2} r-\frac{\hbar r^{3}}{2} \\
& +\frac{1}{2} e^{2 t} \hbar r^{3}-\hbar^{2} r^{3}+e^{2 t} \hbar^{2} r^{3}+3 \hbar t \\
& +\frac{11 \hbar^{2} t}{2}-\frac{1}{4} e^{2 t} \hbar^{2} t+\frac{1}{2} \hbar^{2} r t+\frac{1}{4} e^{2 t} \hbar^{2} r t \\
& -\hbar r^{3} t-2 \hbar^{2} r^{3} t+2 \hbar t^{2}+\frac{19 \hbar^{2} t^{2}}{4} \\
& +\hbar r t^{2}+\frac{5}{4} \hbar^{2} r t^{2}-\hbar r^{3} t^{2}-2 \hbar^{2} r^{3} t^{2},
\end{aligned}
$$$$
\vdots
$$

Then the solutions obtained by HAM are as follows:

$$
\begin{aligned}
\underline{Y}(t, r)= & z_{1,0}(t, r)+z_{1,1}(t, r) \\
& +z_{1,2}(t, r)+z_{1,3}(t, r)+\cdots, \\
\bar{Y}(t, r)= & z_{2,0}(t, r)+z_{2,1}(t, r) \\
& +z_{2,2}(t, r)+z_{2,3}(t, r)+\cdots, \\
\underline{G}(t, r)= & z_{3,0}(t, r)+z_{3,1}(t, r) \\
& +z_{3,2}(t, r)+z_{3,3}(t, r)+\cdots, \\
\bar{G}(t, r)= & z_{4,0}(t, r)+z_{4,1}(t, r) \\
& +z_{4,2}(t, r)+z_{4,3}(t, r)+\cdots,
\end{aligned}
$$

and Figures 7, 8, 9, and 10 show the $\hbar$-meshes of $\left(\partial^{3} / \partial t^{3}\right) \underline{Y}_{10}(0, r),\left(\partial^{3} / \partial t^{3}\right) \bar{Y}_{10}(0, r),(\partial / \partial t) \underline{G}_{10}(0.1, r)$, and $(\partial / \partial t) \bar{G}_{10}(0.1, r)$ respectively. Also, Figures $11,12,13$, and 14 exhibit their related Contour plots.

By minimizing the residual error, we find that the best choices for auxiliary parameter to approximate of $\underline{Y}_{15}(t, r)$, $\bar{Y}_{15}(t, r), \underline{G}_{15}(t, r)$, and $\bar{G}_{15}(t, r)$ are

$$
\begin{array}{ll}
\hbar=-0.972656, & \hbar=-0.97265, \\
\hbar=-0.972969, & \hbar=-0.972964,
\end{array}
$$

respectively. Absolute errors for the 15th order approximation by HAM for $\underline{Y}_{n}(t, r)$ and $\bar{Y}_{n}(t, r), \underline{G}_{n}(t, r), \bar{G}_{n}(t, r)$ are plotted in Figures 15, 16, 17, and 18, respectively.

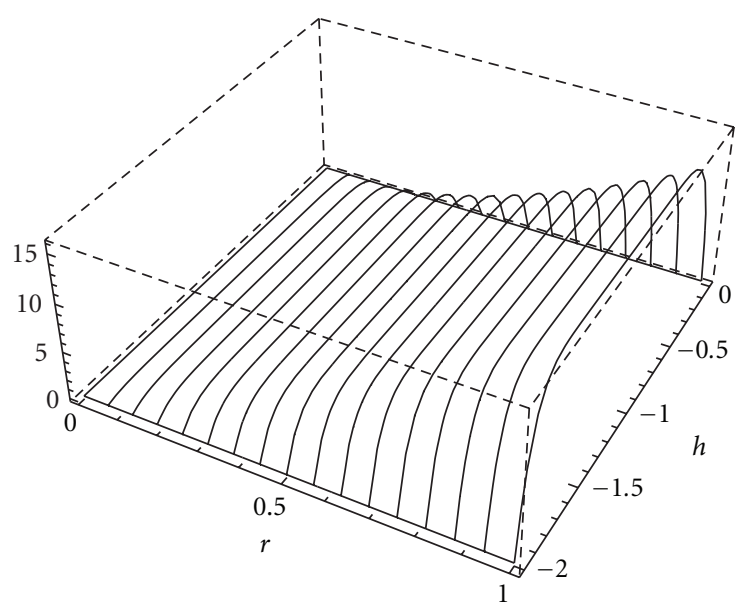

Figure $7: \hbar$-mesh of $\left(\partial^{3} / \partial t^{3}\right) \underline{Y}_{10}(0, r)$.

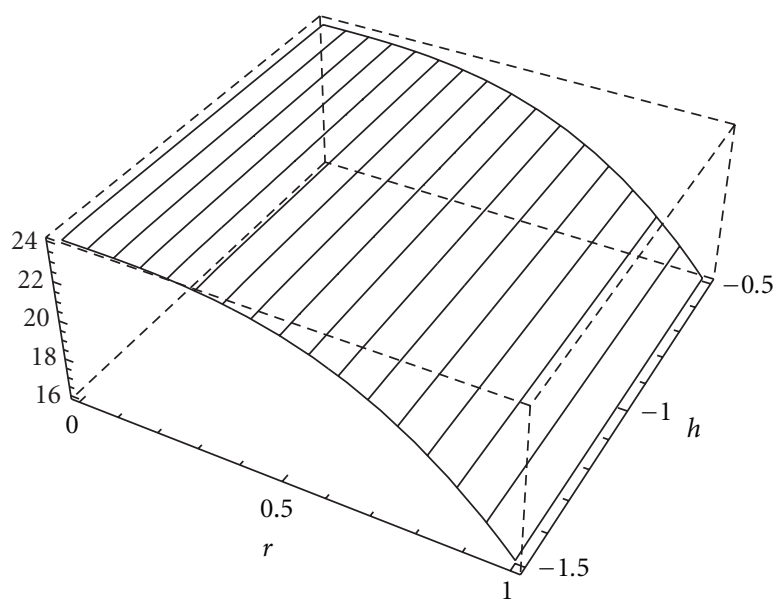

Figure $8: \hbar$-mesh of $\left(\partial^{3} / \partial t^{3}\right) \bar{Y}_{10}(0, r)$

Example 9. Let us consider the first-order system of fuzzy differential equation

$$
\begin{gathered}
Y^{\prime}-G=\left((-2 t r+2 t+2 r+3) e^{2 t},(2 t r-2 t-2 r+7) e^{2 t}\right), \\
Y+G^{\prime}=\left((-r t+t+3 r+2) e^{2 t},(r t-t-3 r+8) e^{2 t}\right), \\
Y(0)=(2+r, 4-r), \quad G(0)=(r, 2-r), \\
t \in[0,1] .
\end{gathered}
$$

The exact solution is as follows:

$$
\begin{gathered}
Y(t, r)=\left((2+r) e^{2 t}+(1-r) t e^{2 t},(4-r) e^{2 t}+(r-1) t e^{2 t}\right), \\
G(t, r)=\left(r e^{2 t},(2-r) e^{2 t}\right) .
\end{gathered}
$$




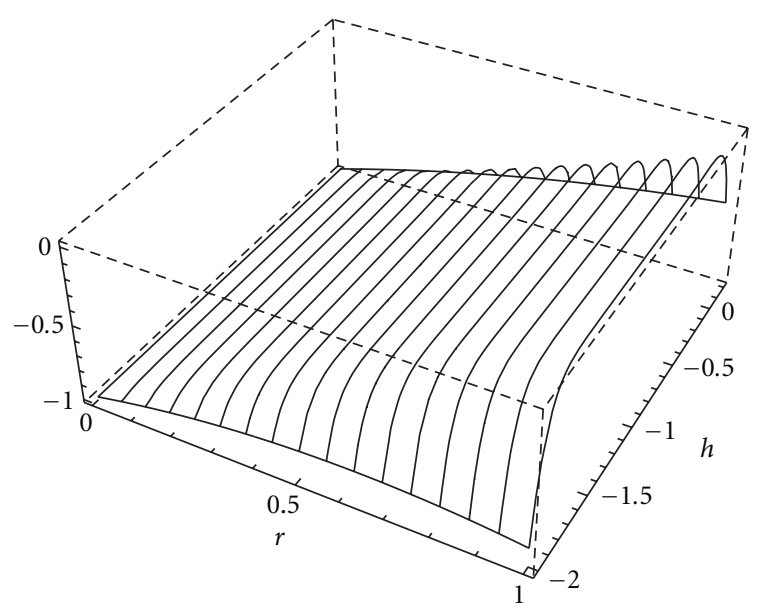

Figure 9: $\hbar$-mesh of $(\partial / \partial t) \underline{G}_{10}(0.1, r)$.



Figure 10: $\hbar$-mesh of $(\partial / \partial t) \bar{G}_{10}(0.1, r)$.

We may replace (67) by the equivalent system

$$
\begin{aligned}
& \underline{Y}^{\prime}-\bar{G}=(-2 t r+2 t+2 r+3) e^{2 t}, \\
& \bar{Y}^{\prime}-\underline{G}=(2 t r-2 t-2 r+7) e^{2 t}, \\
& \underline{Y}+\underline{G}^{\prime}=(-r t+t+3 r+2) e^{2 t}, \\
& \bar{Y}+\bar{G}^{\prime}=\left((r t-t-3 r+8) e^{2 t}\right), \\
& \underline{Y}(0, r)=2+r, \quad \bar{Y}(0, r)=4-r, \\
& \underline{G}(0, r)=r, \quad \bar{G}(0, r)=2-r .
\end{aligned}
$$

We choose the initial guesses of system (69) as follows:

$$
\begin{aligned}
& \underline{Y}_{0}=2+r, \quad \bar{Y}_{0}=4-r, \\
& \underline{G}_{0}=r, \quad \bar{G}_{0}=2-r,
\end{aligned}
$$

and the auxiliary linear operators

$$
\mathcal{L}_{i}\left[\phi_{i}(t, r ; q)\right]=\frac{\partial \phi_{i}(t, r ; q)}{\partial t}, \quad i=1,2,3,4,
$$

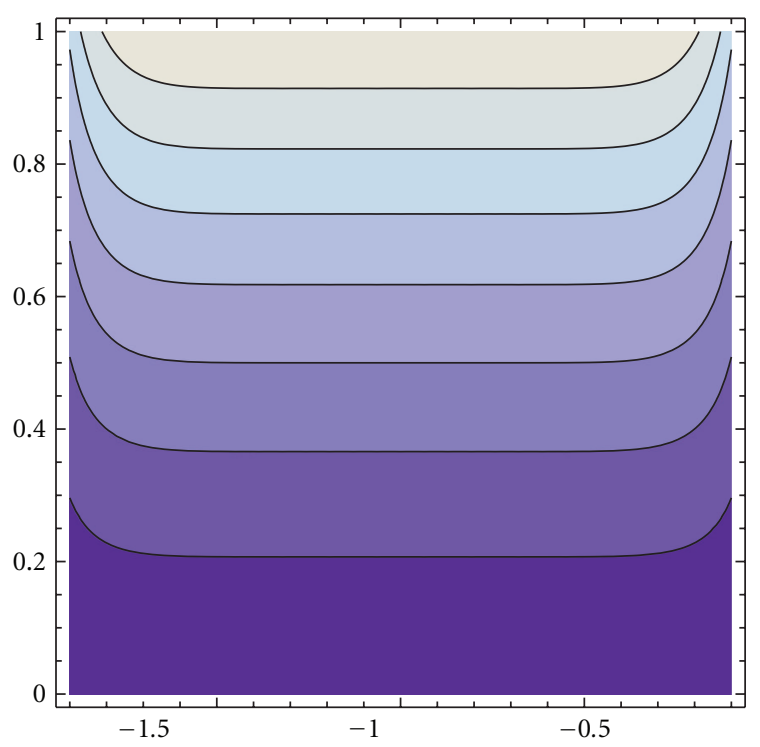

Figure 11: Contour plot of $\left(\partial^{3} / \partial t^{3}\right) \underline{Y}_{10}(0, r)$.

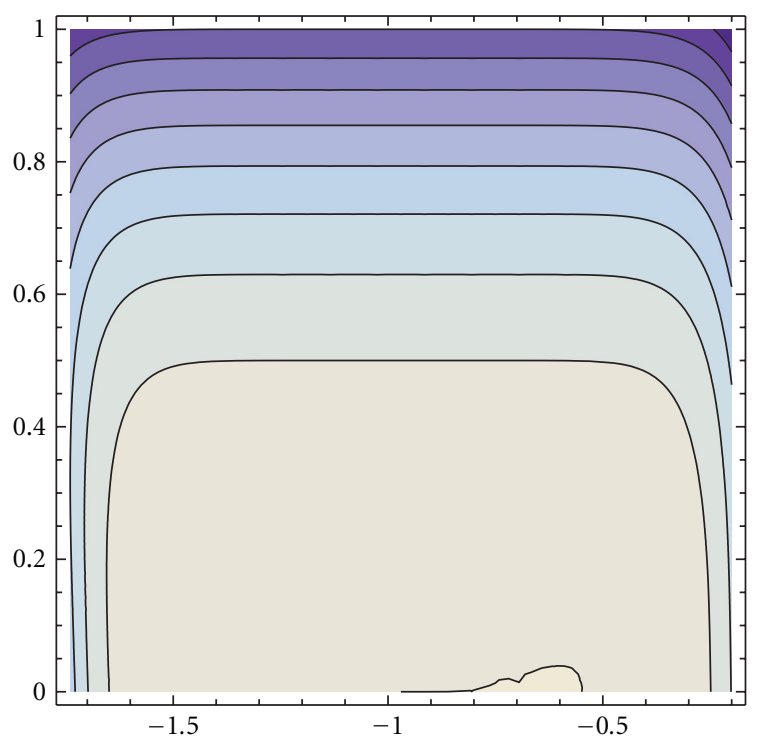

FIGURE 12: Contour plot of $\left(\partial^{3} / \partial t^{3}\right) \bar{Y}_{10}(0, r)$.

with the properties

$$
\mathcal{L}_{i}\left[c_{i}\right]=0, \quad i=1,2,3,4,
$$

where $c_{i}(i=1,2,3,4)$ are integral constants, and the nonlinear operators,

$$
\begin{aligned}
\mathcal{N}_{1}\left[\phi_{1}(t, r ; q), \ldots, \phi_{4}(t, r ; q)\right] \\
=\frac{\partial \phi_{1}(t, r ; q)}{\partial t}-\phi_{4}(t, r ; q) \\
\quad-(-2 t r+2 t+2 r+3) e^{2 t}
\end{aligned}
$$




$$
\begin{array}{r}
\mathcal{N}_{2}\left[\phi_{1}(t, r ; q), \ldots, \phi_{4}(t, r ; q)\right] \\
=\frac{\partial \phi_{2}(t, r ; q)}{\partial t}-\phi_{3}(t, r ; q) \\
-(2 t r-2 t-2 r+7) e^{2 t}, \\
\mathcal{N}_{3}\left[\phi_{1}(t, r ; q), \ldots, \phi_{4}(t, r ; q)\right] \\
=\frac{\partial \phi_{3}(t, r ; q)}{\partial t}+\phi_{1}(t, r ; q) \\
-(-r t+t+3 r+2) e^{2 t}, \\
\mathcal{N}_{4}\left[\phi_{1}(t, r ; q), \ldots, \phi_{4}(t, r ; q)\right] \\
=\frac{\partial \phi_{4}(t, r ; q)}{\partial t}+\phi_{2}(t, r ; q) \\
-(r t-t-3 r+8) e^{2 t} .
\end{array}
$$

The $m$ th order deformation equations are

$$
\begin{array}{r}
\mathcal{L}_{i}\left[z_{i, m}(t, r)-\chi_{m} z_{i, m-1}(t, r)\right]=\hbar \mathcal{R}_{i, m}\left(\vec{z}_{1, m-1}, \ldots, \vec{z}_{4, m-1}\right), \\
i=1,2,3,4,
\end{array}
$$

with the initial conditions

$$
z_{i, m}(0, r)=0, \quad i=1,2,3,4,
$$

where

$$
\begin{aligned}
& \mathcal{R}_{1, m}\left(\vec{z}_{1, m-1}, \ldots, \vec{z}_{4, m-1}\right) \\
&= \frac{\partial z_{1, m-1}}{\partial t}-z_{4, m-1} \\
&-\left(1-\chi_{m}\right)(-2 t r+2 t+2 r+3) e^{2 t}, \\
& \mathcal{R}_{2, m}\left(\vec{z}_{1, m-1}, \ldots, \vec{z}_{4, m-1}\right) \\
&= \frac{\partial z_{2, m-1}}{\partial t}-z_{3, m-1} \\
&-\left(1-\chi_{m}\right)(2 t r-2 t-2 r+7) e^{2 t}, \\
& \mathcal{R}_{3, m}\left(\vec{z}_{1, m-1}, \ldots, \vec{z}_{4, m-1}\right) \\
&=\frac{\partial z_{3, m-1}}{\partial t}+z_{1, m-1} \\
&-\left(1-\chi_{m}\right)(-r t+t+3 r+2) e^{2 t}, \\
& \mathcal{R}_{4, m}\left(\vec{z}_{1, m-1}, \ldots, \vec{z}_{4, m-1}\right) \\
&=\frac{\partial z_{4, m-1}}{\partial t}+z_{2, m-1} \\
&-\left(1-\chi_{m}\right)(r t-t-3 r+8) e^{2 t} .
\end{aligned}
$$
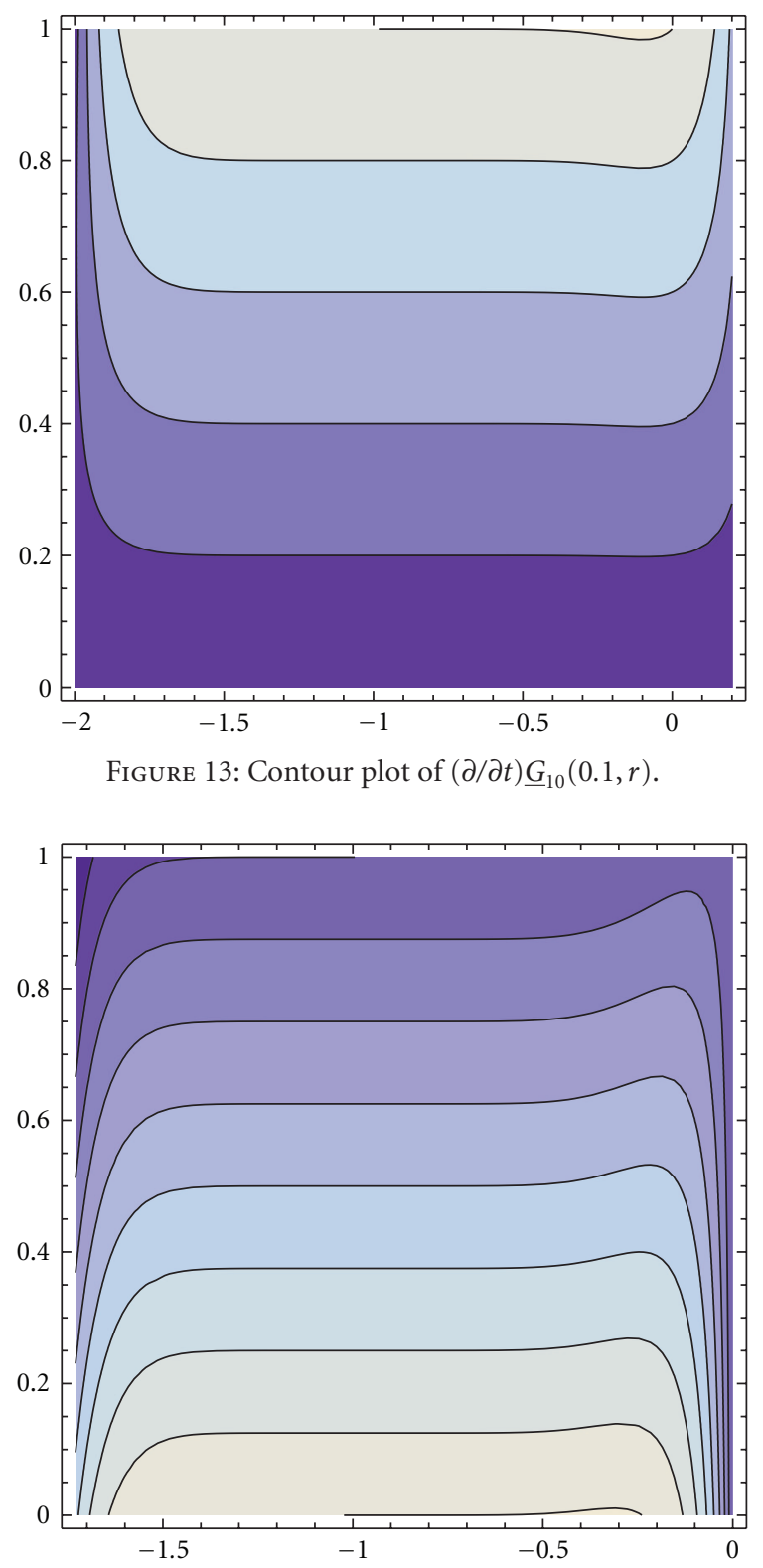

Figure 14: Contour plot of $(\partial / \partial t) \bar{G}_{10}(0.1, r)$.

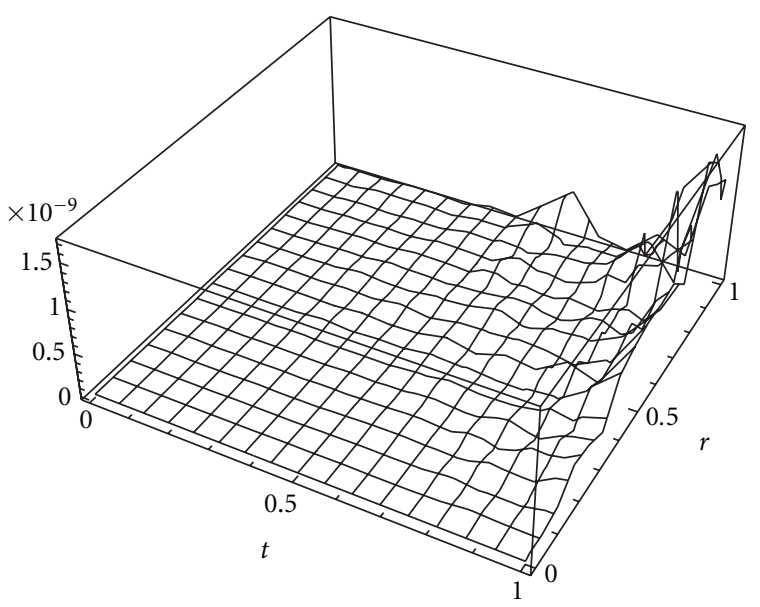

FIGURE 15: Absolute error of $\underline{Y}_{15}(t, r)$ for $\hbar=-0.972656$. 


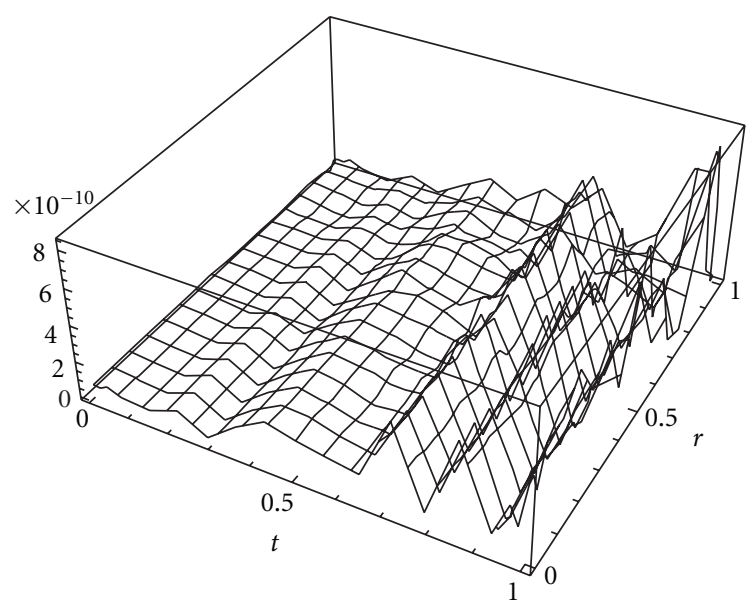

Figure 16: Absolute error of $\bar{Y}_{15}(t, r)$ for $\hbar=-0.97265$.



FIGURE 17: Absolute error of $\underline{G}_{15}(t, r)$ for $\hbar=-0.972969$.

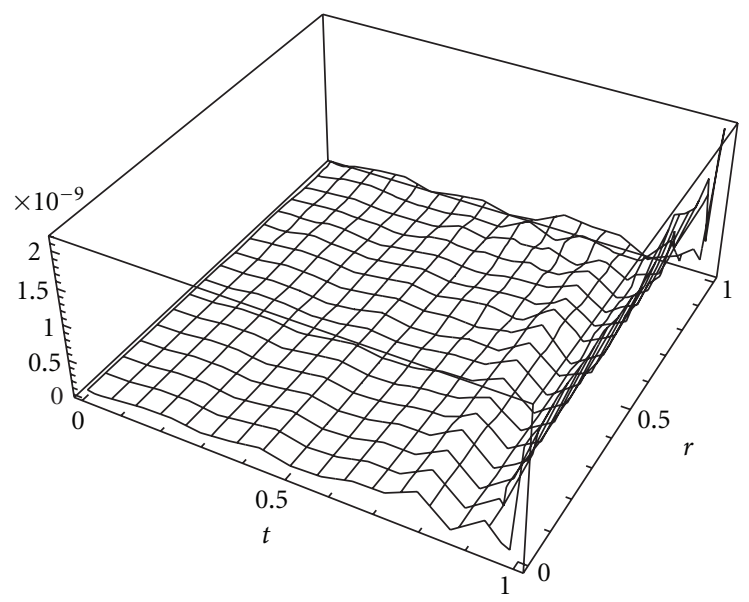

FIgURE 18: Absolute error of $\bar{G}_{15}(t, r)$ for $\hbar=-0.972964$.
Therefore, we recursively obtain

$$
\begin{aligned}
z_{1,1}(t, r)= & \hbar-e^{2 t} \hbar+\frac{3 \hbar r}{2}-\frac{3 e^{2 t} \hbar r}{2}-2 \hbar t \\
& -e^{2 t} \hbar t+\hbar r t+e^{2 t} \hbar r t, \\
z_{1,2}(t, r)= & \hbar-e^{2 t} \hbar-\frac{5 \hbar^{2}}{4}+\frac{5 e^{2 t} \hbar^{2}}{4} \\
& +\frac{3 \hbar r}{2}-\frac{3 e^{2 t} \hbar r}{2}+\frac{5 \hbar^{2} r}{2}-\frac{5 e^{2 t} \hbar^{2} r}{2}-2 \hbar t-e^{2 t} \hbar t \\
& -\frac{25 \hbar^{2} t}{4}-\frac{5 e^{2 t} \hbar^{2} t}{4}+\hbar r t+e^{2 t} \hbar r t \\
& +\frac{11 \hbar^{2} r t}{4}+\frac{5 e^{2 t} \hbar^{2} r t}{4}-2 \hbar^{2} t^{2}+\frac{\hbar^{2} r t^{2}}{2}, \\
z_{2,1}(t, r)= & 4 \hbar-4 e^{2 t} \hbar-\frac{3 \hbar r}{2}+\frac{3 e^{2 t} \hbar r}{2}+e^{2 t} \hbar t \\
& -\hbar r t-e^{2 t} \hbar r t,
\end{aligned}
$$$$
z_{2,2}(t, r)=4 \hbar-4 e^{2 t} \hbar+\frac{15 \hbar^{2}}{4}-\frac{15 e^{2 t} \hbar^{2}}{4}
$$$$
-\frac{3 \hbar r}{2}+\frac{3 e^{2 t} \hbar r}{2}
$$$$
-\frac{5 \hbar^{2} r}{2}+\frac{5 e^{2 t} \hbar^{2} r}{2}+e^{2 t} \hbar t-\frac{3 \hbar^{2} t}{4}
$$$$
+\frac{5 e^{2 t} \hbar^{2} t}{4}-\hbar r t-e^{2 t} \hbar r t
$$$$
-\frac{11 \hbar^{2} r t}{4}-\frac{5 e^{2 t} \hbar^{2} r t}{4}-\hbar^{2} t^{2}-\frac{\hbar^{2} r t^{2}}{2},
$$$$
z_{3,1}(t, r)=\frac{3 \hbar}{4}-\frac{3 e^{2 t} \hbar}{4}+\frac{7 \hbar r}{4}-\frac{7 e^{2 t} \hbar r}{4}+2 \hbar t
$$$$
-\frac{e^{2 t} \hbar t}{2}+\hbar r t+\frac{e^{2 t} \hbar r t}{2}
$$$$
z_{3,2}(t, r)=\frac{3 \hbar}{4}-\frac{3 e^{2 t} \hbar}{4}+\hbar^{2}-e^{2 t} \hbar^{2}+\frac{7 \hbar r}{4}-\frac{7 e^{2 t} \hbar r}{4}
$$$$
+\frac{11 \hbar^{2} r}{4}-\frac{11 e^{2 t} \hbar^{2} r}{4}+2 \hbar t-\frac{e^{2 t} \hbar t}{2}
$$$$
+3 \hbar^{2} t-e^{2 t} \hbar^{2} t+\hbar r t+\frac{e^{2 t} \hbar r t}{2}
$$$$
+\frac{5 \hbar^{2} r t}{2}+e^{2 t} \hbar^{2} r t-\hbar^{2} t^{2}+\frac{\hbar^{2} r t^{2}}{2},
$$$$
z_{4,1}(t, r)=\frac{17 \hbar}{4}-\frac{17 e^{2 t} \hbar}{4}-\frac{7 \hbar r}{4}+\frac{7 e^{2 t} \hbar r}{4}+4 h t
$$$$
+\frac{e^{2 t} \hbar t}{2}-\hbar r t-\frac{e^{2 t} \hbar r t}{2}
$$$$
z_{4,2}(t, r)=\frac{17 \hbar}{4}-\frac{17 e^{2 t} \hbar}{4}
$$$$
+\frac{13 \hbar^{2}}{2}-\frac{13 e^{2 t} \hbar^{2}}{2}-\frac{7 \hbar r}{4}+\frac{7 e^{2 t} \hbar r}{4}
$$$$
-\frac{11 \hbar^{2} r}{4}+\frac{11 e^{2 t} \hbar^{2} r}{4}+4 \hbar t+\frac{e^{2 t} \hbar t}{2}
$$$$
+8 \hbar^{2} t+e^{2 t} \hbar^{2} t-\hbar r t-\frac{e^{2 t} \hbar r t}{2}
$$$$
-\frac{5 \hbar^{2} r t}{2}-e^{2 t} \hbar^{2} r t-\frac{\hbar^{2} r t^{2}}{2},
$$ 


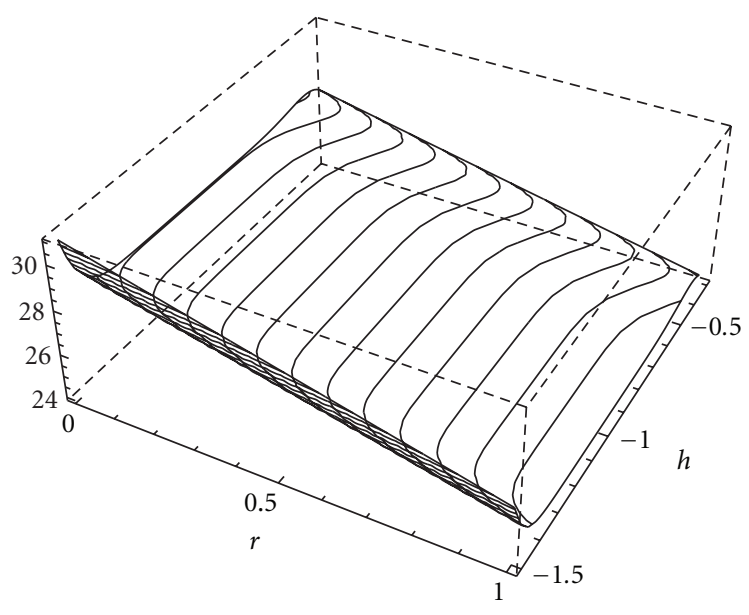

Figure 19: $\hbar$-mesh of $\left(\partial^{3} / \partial t^{3}\right) \underline{Y}_{10}(0, r)$.



Figure 20: $\hbar$-mesh of $\left(\partial^{3} / \partial t^{3}\right) \bar{Y}_{10}(0, r)$.

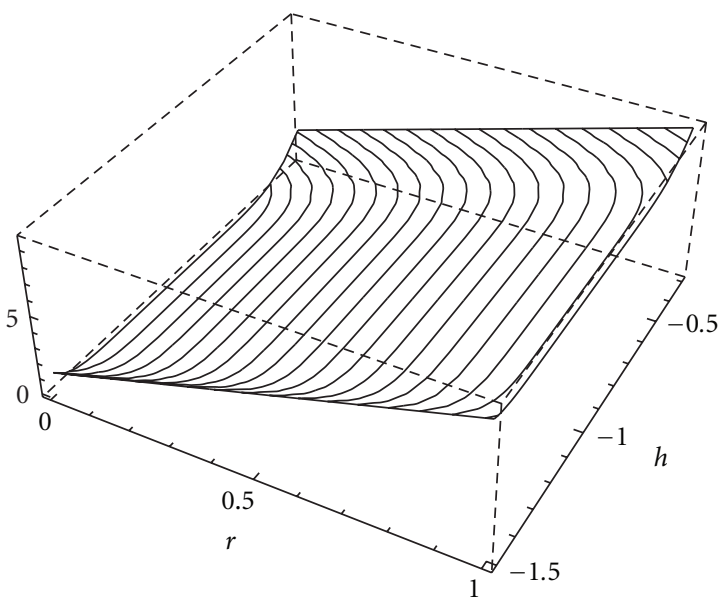

FIGURE 21: $\hbar$-mesh of $\left(\partial^{3} / \partial t^{3}\right) \underline{G}_{10}(0, r)$.



FIGURE 22: $\hbar$-mesh of $\left(\partial^{3} / \partial t^{3}\right) \bar{G}_{10}(0, r)$.

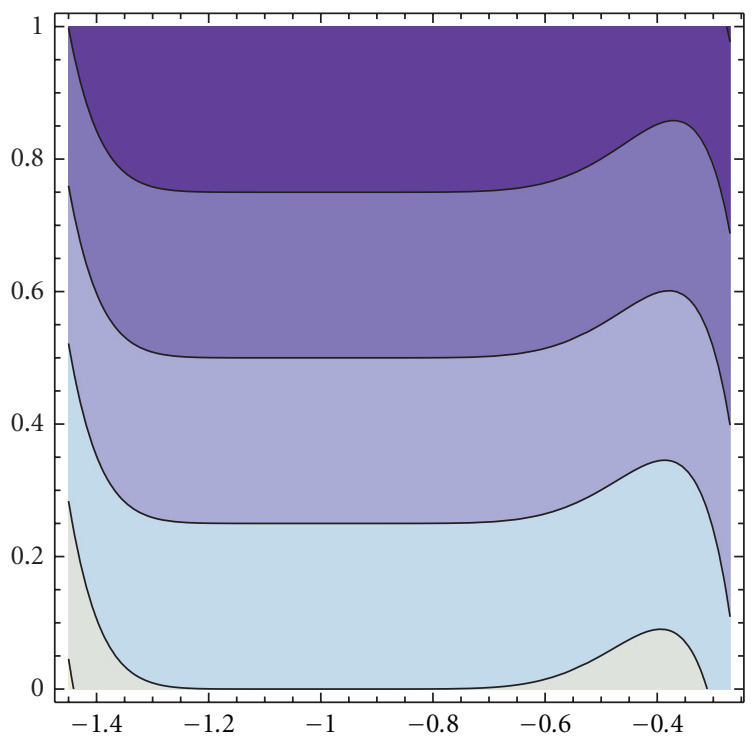

FIGURE 23: Contour plot of $\left(\partial^{3} / \partial t^{3}\right) \underline{Y}_{10}(0, r)$.

Then obtained solutions are as follows:

$$
\begin{aligned}
& \underline{Y}(t, r)=z_{1,0}(t, r)+z_{1,1}(t, r)+z_{1,2}(t, r)+z_{1,3}(t, r)+\cdots, \\
& \bar{Y}(t, r)=z_{2,0}(t, r)+z_{2,1}(t, r)+z_{2,2}(t, r)+z_{2,3}(t, r)+\cdots, \\
& \underline{G}(t, r)=z_{3,0}(t, r)+z_{3,1}(t, r)+z_{3,2}(t, r)+z_{3,3}(t, r)+\cdots, \\
& \bar{G}(t, r)=z_{4,0}(t, r)+z_{4,1}(t, r)+z_{4,2}(t, r)+z_{4,3}(t, r)+\cdots
\end{aligned}
$$

Figures 19, 20, 21, and 22 show the $\hbar$-meshes of $\left(\partial^{3} / \partial t^{3}\right) \underline{Y}_{10}(0, r), \quad\left(\partial^{3} / \partial t^{3}\right) \bar{Y}_{10}(0, r), \quad\left(\partial^{3} / \partial t^{3}\right) \underline{G}_{10}(0, r)$ and $\left(\partial^{3} / \partial t^{3}\right) \bar{G}_{10}(0, r)$, respectively. Also, Figures $23,24,25$, and 26 show the related Contour plots. 




Figure 24: Contour plot of $\left(\partial^{3} / \partial t^{3}\right) \bar{Y}_{10}(0, r)$.



FIgURe 25: Contour plot of $\left(\partial^{3} / \partial t^{3}\right) \underline{G}_{10}(0, r)$.

Best choices for auxiliary parameter $\hbar$ to approximate $\underline{Y}_{15}(t, r), \bar{Y}_{15}(t, r), \underline{G}_{15}(t, r)$, and $\bar{G}_{15}(t, r)$ from minimizing the residual errors are

$$
\begin{aligned}
& \hbar=-0.977581, \quad \hbar=-0.968521, \\
& \hbar=-1.01756, \quad \hbar=-0.978537,
\end{aligned}
$$

respectively. Finally, absolute errors for the 15th order approximation by HAM for $\underline{Y}_{n}(t, r), \bar{Y}_{n}(t, r), \underline{G}_{n}(t, r)$ and $\bar{G}_{n}(t, r)$, are plotted in Figures 27, 28, 29, and 30.

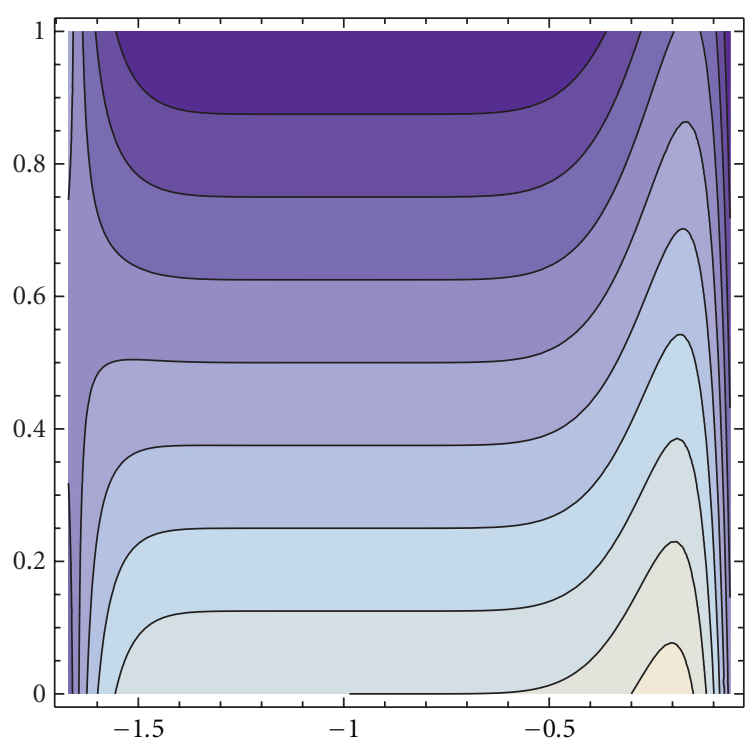

Figure 26: Contour plot of $\left(\partial^{3} / \partial t^{3}\right) \bar{G}_{10}(0, r)$.

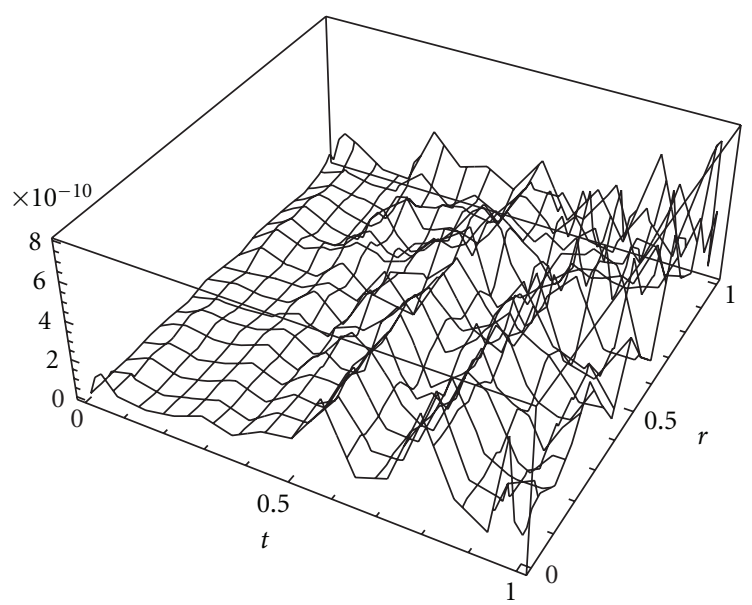

Figure 27: Absolute error of $\underline{Y}_{15}(t, r)$ for $\hbar=-0.977581$.

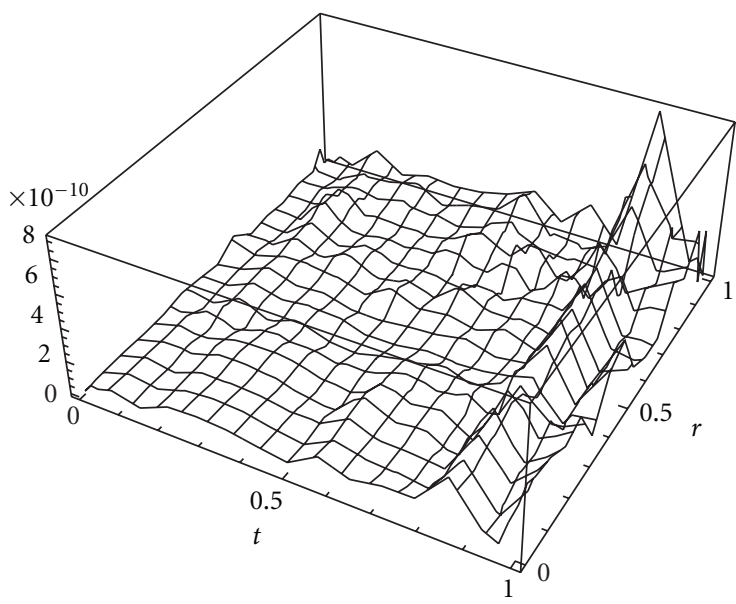

FIgURE 28: Absolute error of $\bar{Y}_{15}(t, r)$ for $\hbar=-0.968521$. 


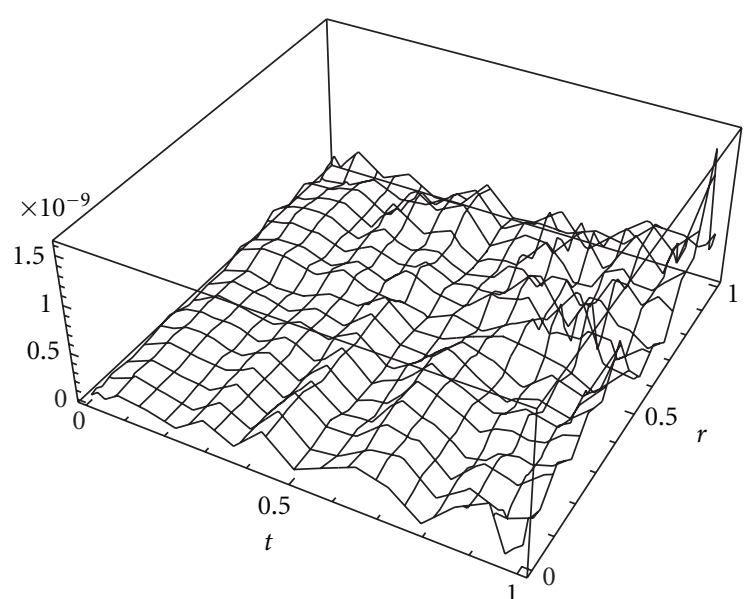

FIGURE 29: Absolute error of $\underline{G}_{15}(t, r)$ for $\hbar=-1.01756$.

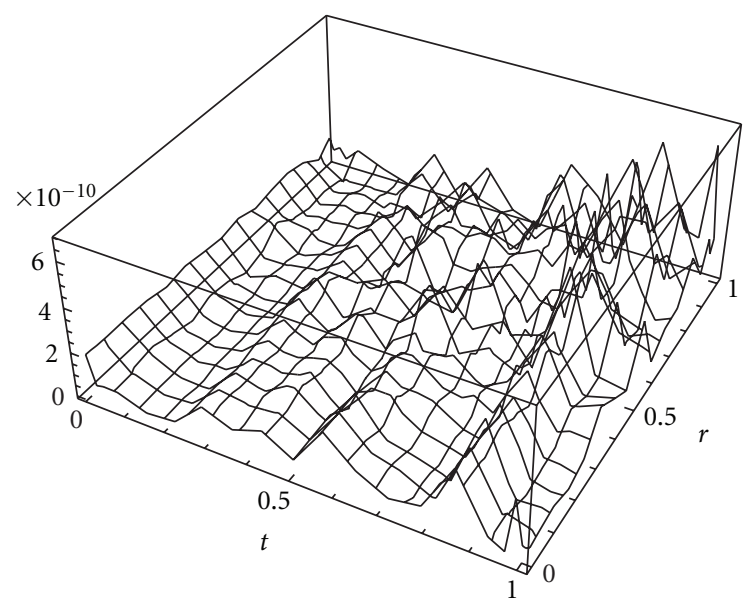

FigURe 30: Absolute error of $\bar{G}_{15}(t, r)$ for $\hbar=-0.978537$.

\section{Conclusion}

In this paper, homotopy analysis method has been implemented to derive approximate analytical solutions for the system of fuzzy differential equations. Obtained results show that we can control the convergence region of HAM series solution by the auxiliary parameter $\hbar$. The concept of traditional $h$-curves has been generalized to $\hbar$-meshes and then contour plot firstly has been introduced in HAM. Convergence theorem and given illustrative examples show the efficiency and accuracy of the HAM.

\section{Acknowledgments}

The respected anonymous referees have carefully reviewed this paper. As a result of their careful analysis, our paper has been improved. The authors would like to express their thankfulness to them for their helpful constructive comments.

\section{References}

[1] L. A. Zadeh, "Fuzzy sets," Information and Control, vol. 8, no. 3, pp. 338-353, 1965.

[2] S. L. Chang and L. A. Zadeh, "On fuzzy mapping and control," IEEE Transactions on Systems, Man and Cybernetics, vol. 2, no. 1, pp. 30-34, 1972.

[3] D. Dubois and H. Prade, "Towards fuzzy differential calculus-part 3: differentiation," Fuzzy Sets and Systems, vol. 8, no. 3, pp. 225-233, 1982.

[4] M. L. Puri and D. Ralescu, "Differentials of fuzzy functions," Journal of Mathematical Analysis and Applications, vol. 91, pp. 321-325, 1983.

[5] R. Goetschel and W. Voxman, "Elementary fuzzy calculus," Fuzzy Sets and Systems, vol. 18, no. 1, pp. 31-43, 1986.

[6] O. Kaleva, "Fuzzy differential equations," Fuzzy Sets and Systems, vol. 24, no. 3, pp. 301-317, 1987.

[7] O. Kaleva, "The cauchy problem for fuzzy differential equations," Fuzzy Sets and Systems, vol. 35, no. 3, pp. 389-396, 1990.

[8] S. Seikkala, "On the fuzzy initial value problem," Fuzzy Sets and Systems, vol. 24, no. 3, pp. 319-330, 1987.

[9] S. J. Liao, The proposed homotopy analysis technique for the solution of nonlinear problems [Ph.D. thesis], Shanghai Jiao Tong University, 1992.

[10] S. j. Liao, Beyond Perturbation: Introduction to the Homotopy Analysis Method, CRC Press, Chapman Hall, Boca Raton, Fla, USA, 2003.

[11] Z. Akbarzadeh Ghanaie and M. Mohseni Moghadam, "Solving fuzzy differential equations by Runge-Kutta method," The Journal of Mathematics and Computer Science, vol. 2, no. 2, pp. 208-221, 2011.

[12] T. Allahviranloo, E. Ahmady, and N. Ahmady, "Nth-order fuzzy linear differential equations," Information Sciences, vol. 178, no. 5, pp. 1309-1324, 2008.

[13] T. Allahviranloo, N. Ahmady, and E. Ahmady, "Numerical solution of fuzzy differential equations by predictor-corrector method," Information Sciences, vol. 177, no. 7, pp. 1633-1647, 2007.

[14] E. Babolian, H. Sadeghi, and S. Javadi, "Numerically solution of fuzzy differential equations by Adomian method," Applied Mathematics and Computation, vol. 149, no. 2, pp. 547-557, 2004.

[15] M. Friedman, M. Ma, and A. Kandel, "Numerical solutions of fuzzy differential and integral equations," Fuzzy Sets and Systems, vol. 106, no. 1, pp. 35-48, 1999.

[16] A. Sami Bataineh, M. S. M. Noorani, and I. Hashim, "Solving systems of ODEs by homotopy analysis method," Communications in Nonlinear Science and Numerical Simulation, vol. 13, no. 10, pp. 2060-2070, 2008.

[17] A. Sami Bataineh, M. S. M. Noorani, and I. Hashim, "Approximate analytical solutions of systems of PDEs by homotopy analysis method," Computers and Mathematics with Applications, vol. 55, no. 12, pp. 2913-2923, 2008.

[18] S. Abbasbandy, "Soliton solutions for the Fitzhugh-Nagumo equation with the homotopy analysis method," Applied Mathematical Modelling, vol. 32, no. 12, pp. 2706-2714, 2008.

[19] M. Turkyilmazoglu, "Numerical and analytical solutions for the flow and heat transfer near the equator of an MHD boundary layer over a porous rotating sphere," International Journal of Thermal Sciences, vol. 50, no. 5, pp. 831-842, 2011.

[20] A. Elsaid, "Homotopy analysis method for solving a class of fractional partial differential equations," Communications in 
Nonlinear Science and Numerical Simulation, vol. 16, no. 9, pp. 3655-3664, 2011.

[21] S. Xinhui, Z. Liancun, Z. Xinxin, and Y. Jianhong, "Homotopy analysis method for the heat transfer in a asymmetric porous channel with an expanding or contracting wall," Applied Mathematical Modelling, vol. 35, no. 9, pp. 4321-4329, 2011.

[22] S. Nadeem, T. Hayat, S. Abbasbandy, and M. Ali, "Effects of partial slip on a fourth-grade fluid with variable viscosity: an analytic solution," Nonlinear Analysis: Real World Applications, vol. 11, no. 2, pp. 856-868, 2010.

[23] M. S. Hashemi, M. K. Mirnia, and S. Shahmorad, "Solving fuzzy linear systems by using the schur complement when coefficient matrix is an M-matrix," Iranian Journal of Fuzzy Systems, vol. 5, no. 3, pp. 15-29, 2008. 

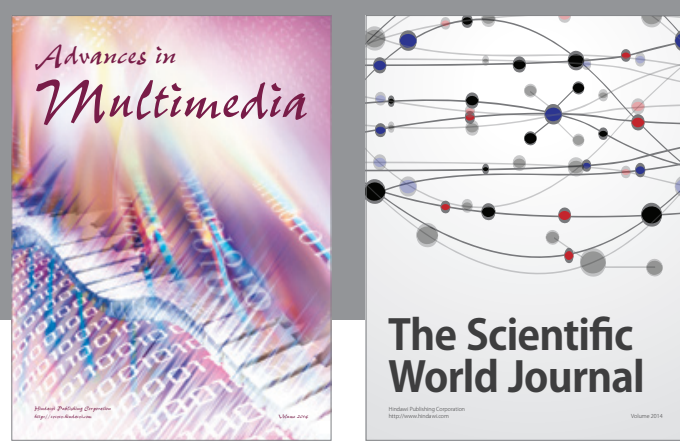

The Scientific World Journal
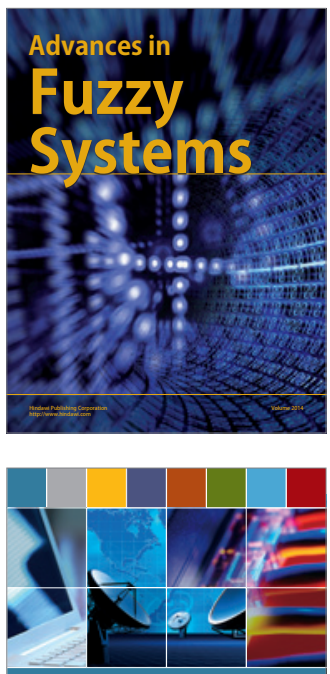

Computer Networks and Communications
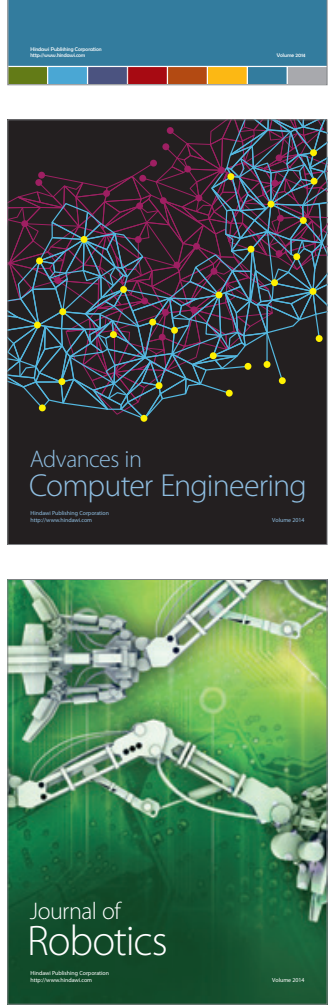
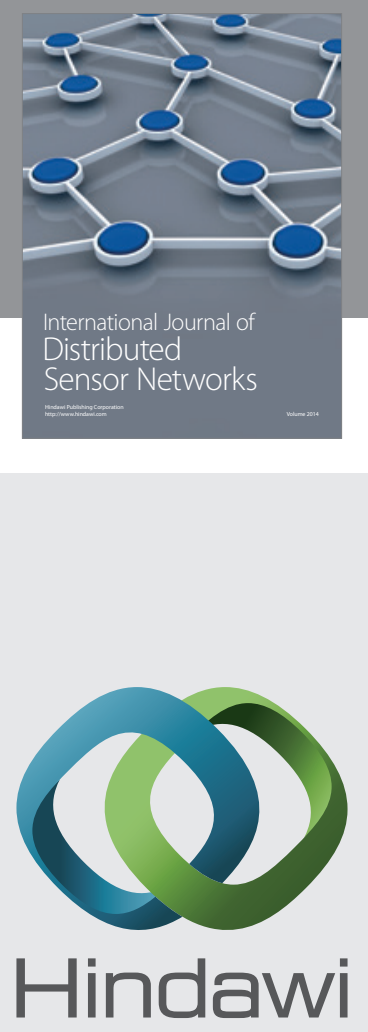

Submit your manuscripts at

http://www.hindawi.com
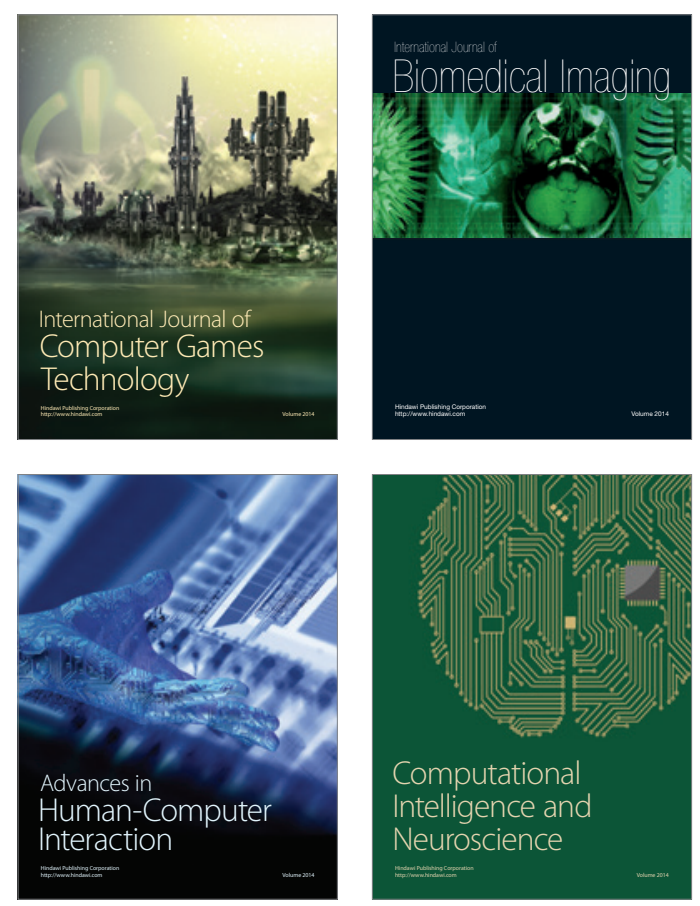
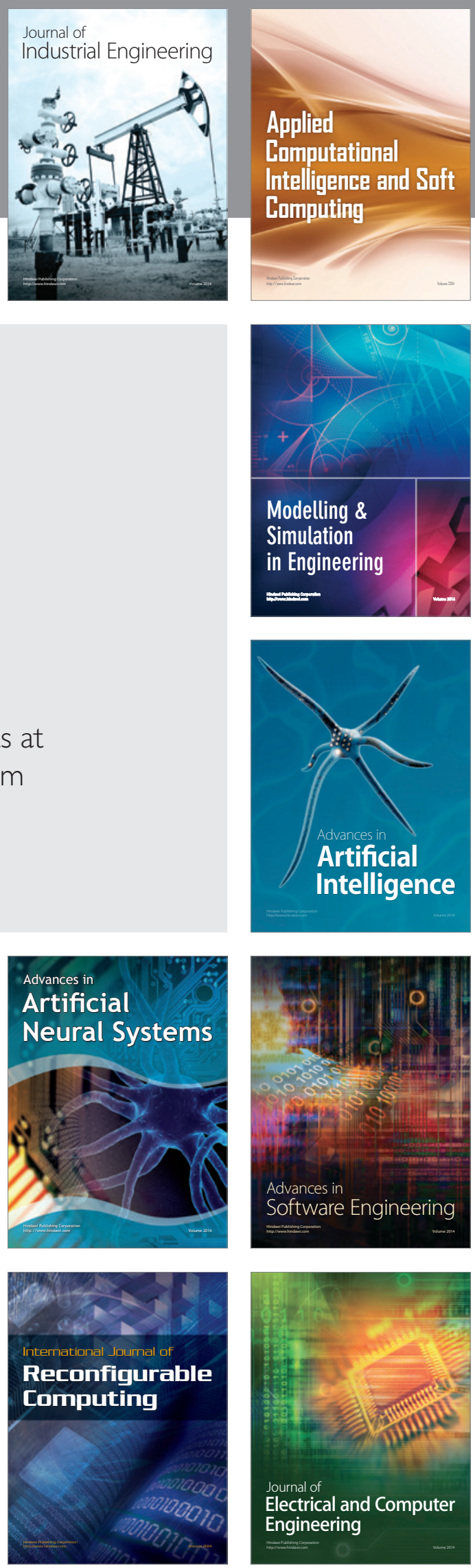